\title{
ON THE QUASI-UNCONDITIONAL STABILITY OF BDF-ADI SOLVERS FOR THE COMPRESSIBLE NAVIER-STOKES EQUATIONS AND RELATED LINEAR PROBLEMS*
}

\author{
OSCAR P. BRUNO ${ }^{\dagger}$ AND MAX CUBILLOS ${ }^{\dagger}$
}

\begin{abstract}
The companion paper "Higher-order in time quasi-unconditionally stable ADI solvers for the compressible Navier-Stokes equations in 2D and 3D curvilinear domains," which is referred to as Part I in what follows, introduces ADI (alternating direction implicit) solvers of higher orders of temporal accuracy (orders $s=2$ to 6 ) for the compressible Navier-Stokes equations in two- and threedimensional space. The proposed methodology employs the backward differentiation formulae (BDF) together with a quasilinear-like formulation, high-order extrapolation for nonlinear components, and the Douglas-Gunn splitting. A variety of numerical results presented in Part I demonstrate in practice the theoretical convergence rates enjoyed by these algorithms, as well as their excellent accuracy and stability properties for a wide range of Reynolds numbers. In particular, the proposed schemes enjoy a certain property of "quasi-unconditional stability": for small enough (problemdependent) fixed values of the timestep $\Delta t$, these algorithms are stable for arbitrarily fine spatial discretizations. The present contribution presents a mathematical basis for the observed performance of these algorithms. Short of providing stability theorems for the full Navier-Stokes BDF-ADI solvers, this paper puts forth a number of stability proofs for BDF-ADI schemes as well as some related unsplit BDF schemes for a variety of related linear model problems in one, two, and three spatial dimensions. These include proofs of quasi-unconditional stability for unsplit BDF schemes of orders $2 \leq s \leq 6$, and even a proof of a form of unconditional stability for two-dimensional BDF-ADI schemes of order 2 for both convection and diffusion problems. Additionally, a set of numerical tests presented in this paper for the compressible Navier-Stokes equation indicate that quasi-unconditional stability carries over to the fully nonlinear context.
\end{abstract}

Key words. Navier-Stokes, quasi-unconditional stability, high-order, ADI, BDF, unconditional stability

AMS subject classifications. 65M12, 65M20,65M70

DOI. $10.1137 / 15 \mathrm{M} 1042279$

1. Introduction. The companion paper [4], which is referred to as Part I in what follows, introduces ADI (alternating direction implicit) solvers of higher orders of time accuracy (orders $s=2$ to 6 ) for the compressible Navier-Stokes equations in two- and three-dimensional curvilinear domains. Implicit solvers, even of ADI type, are generally more expensive per timestep, for a given spatial discretization, than explicit solvers, but use of efficient implicit solvers can be advantageous whenever the timestep restrictions imposed by the mesh spacing $h$ are too severe. The proposed methodology employs the BDF (backward differentiation formulae) multistep ODE solvers (which are known for their robust stability properties) together with a quasilinear-like formulation and high-order extrapolation for nonlinear components (which gives rise to a linear problem that can be solved efficiently by means of standard linear algebra solvers) and the Douglas-Gunn splitting (an ADI strategy that greatly simplifies the treatment of boundary conditions while retaining the order of time accuracy of the solver).

* Received by the editors October 2, 2015; accepted for publication (in revised form) December 21, 2016; published electronically April 18, 2017.

http://www.siam.org/journals/sinum/55-2/M104227.html

Funding: The work of the first author was supported by AFOSR, NSF and an NSSEFF Vannevar Bush Fellowship under contracts FA9550-15-1-0043, DMS-1411876 and N00014-16-1-2808.

${ }^{\dagger}$ Applied and Computational Mathematics, Caltech, Pasadena, CA, 91125 (obruno@caltech.edu, max.cubillos@gmail.com). 


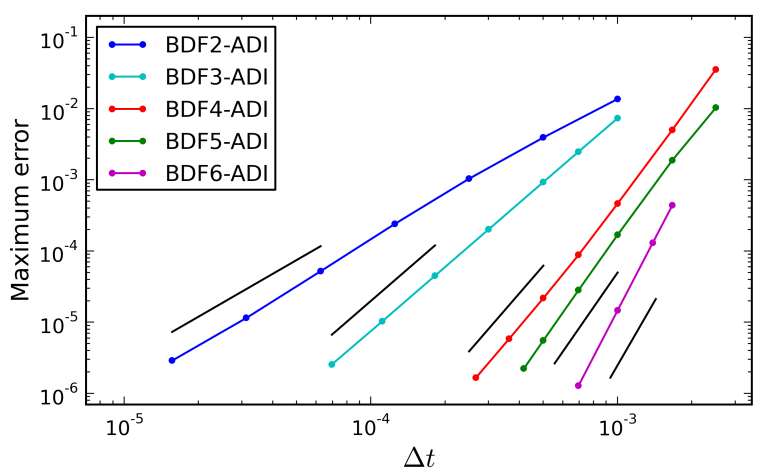

FIG. 1. Temporal convergence of the three-dimensional BDF-ADI Navier-Stokes solvers of orders $s=2, \ldots, 6$ in a curvilinear domain; additional details concerning the high-order convergence of these methods can be found in [4, section 6]. The black lines represent actual slopes of orders 2 (leftmost) through 6 (rightmost).

As discussed in Part I, the proposed BDF-ADI solvers are the first ADI-based Navier-Stokes solvers for which high-order time accuracy has been demonstrated (see Figure 1). In spite of the nominal second order of time accuracy inherent in the celebrated Beam and Warming method [1] (cf. also $[2,16]$ ), previous ADI solvers for the Navier-Stokes equations have not demonstrated time convergence of orders higher than 1 under general nonperiodic physical boundary conditions. Part I demonstrates the properties of the proposed schemes by means of a variety of numerical experiments; the present paper, in turn, provides a theoretical basis for the observed algorithmic stability traits. Short of providing stability theorems for the full BDF-ADI NavierStokes solvers, this paper puts forth proofs of a form of unconditional stability and quasi-unconditional stability (Definition 3.2 below) for BDF-ADI schemes as well as some associated unsplit BDF schemes, for a variety of related linear model problems in one, two, and three spatial dimensions (detailed below in this section), and for schemes of orders $2 \leq s \leq 6$ of temporal accuracy. Further, a variety of numerical tests presented in section 6 indicate that the property of quasi-unconditional stability carries over to the BDF-ADI solvers for the fully nonlinear Navier-Stokes equations.

The BDF-ADI methodology mentioned above can be applied in conjunction with a variety of spatial discretizations. For definiteness, in this contribution attention is restricted to Chebyshev, Legendre, and Fourier spectral spatial approximations. The resulting one-dimensional boundary value problems arising from these discretizations involve full matrices which generally cannot be inverted efficiently by means of a direct solver. However, by relying on fast transforms these systems can be solved effectively on the basis of the GMRES iterative solver; details in these regards are presented in Part I. Additionally, as detailed in that reference, in order to ensure stability for the fully nonlinear Navier-Stokes equations a mild spectral filter is used.

Perhaps the existence of Dahlquist's second barrier may explain the widespread use of implicit methods of orders less than or equal to 2 in the present context (such as backward Euler, the trapezoidal rule, and BDF2, all of which are A-stable), and the virtual absence of implicit methods of orders higher than two - despite the widespread use of the fourth-order Runge-Kutta and Adams-Bashforth explicit counterparts. Clearly, in any case, A-stability is not necessary for all problems - for example, any method whose stability region contains the negative real axis (such as the BDF methods of orders 2 to 6 ) generally results in an unconditionally stable solver for the heat 
equation. A number of important questions thus arise: Are the stability constraints of all higher-order implicit methods too stringent to be useful in the Navier-Stokes context? How close to unconditionally stable can a Navier-Stokes solver be whose temporal order of accuracy is higher than 2 ?

(Attempts to address these questions have lead to semi-implicit Navier-Stokes solvers based in part on the implicit (multistage) Runge-Kutta (RK) scheme: the restriction on the order of accuracy for multistage algorithms is given by a less-stringent generalized version of Dahlquist's second barrier known as the Daniel-Moore conjecture, which states that the order of accuracy of an A-stable method with a number $s$ of implicit stages cannot be larger than $2 s$. In spite of the potential advantages provided by the RK scheme, however, we are not aware of any RK-based NavierStokes implementations for which stability has been demonstrated in practice for a fixed timestep as the meshsize tends to zero.)

Clear answers to these questions are not available in the extant literature; the present work seeks to advance the theoretical understanding in these regards. To illustrate the present state of the art concerning such matters we mention the 2002 reference [3], which compares various implicit methods for the Navier-Stokes equations, where we read, "Practical experience indicates that large-scale engineering computations are seldom stable if run with BDF4. The BDF3 scheme, with its smaller regions of instability, is often stable but diverges for certain problems and some spatial operators. Thus, a reasonable practitioner might use the BDF2 scheme exclusively for large-scale computations." It must be noted, however, that neither the article [3] nor the references it cites investigate in detail the stability restrictions associated with the BDF methods order $s \geq 2$, either theoretically or experimentally. But higher-order methods can be useful: as demonstrated in Part I, methods of order higher than 2 give rise to very significant advantages for certain classes of problems - especially for large-scale computations for which the temporal dispersion inherent in low-order approaches would make it necessary to use inordinately small timesteps.

The recent 2015 article [7], in turn, presents applications of the BDF scheme up to third order of time accuracy in a finite element context for the incompressible Navier-Stokes equations with turbulence modeling. This contribution does not discuss stability restrictions for the third order solver, and, in fact, it only presents numerical examples resulting from use of BDF1 and BDF2. The 2010 contribution [10], which considers a three-dimensional advection-diffusion equation, presents various ADI-type schemes, one of which is based on BDF3. The BDF3 stability analysis in that paper, however, is restricted to the purely diffusive case.

This paper is organized as follows: Section 2 presents a brief derivation of the BDF-ADI method for the two-dimensional pressure-free momentum equation. (A derivation for the full Navier-Stokes equations in a general dimension $d \geq 2$ is given in Part I, but the specialized derivation presented here may prove valuable in view of its relative simplicity.) Section 3 then briefly reviews relevant notions from classical stability theory as well as the concept of quasi-unconditional stability introduced in Part I. Section 4 presents theorems of (classical) unconditional stability for twodimensional BDF-ADI schemes of order $s=2$ specialized to the linear constant coefficient periodic advection equation as well as the linear constant coefficient periodic and nonperiodic parabolic equations. In order to streamline the presentation, the proofs of these results are deferred to Appendices A and B. Considering constant coefficient advection-diffusion equations in one, two, and three spatial dimensions, further, section 5 presents quasi-unconditional stability proofs for the non-ADI BDF methods of orders $s=2$ to 6 along with comparisons of the stability constraints arising from these 
BDF solvers and the commonly-used explicit Adams-Bashforth solvers of orders 3 and 4. Section 6 provides numerical tests that indicate that the BDF-ADI methods of orders $s=2$ to 6 for the the full three-dimensional Navier-Stokes equations also enjoy the property of quasi-unconditional stability; a wide variety of additional numerical experiments are presented in Part I. Section 7, finally, presents a few concluding remarks.

2. The BDF-ADI scheme. In this section we present a derivation of the BDF-ADI scheme in a somewhat simplified context, restricting attention to the twodimensional pressure-free momentum equation

$$
\mathbf{u}_{t}+\mathbf{u} \cdot \nabla \mathbf{u}=\mu\left(\Delta \mathbf{u}+\frac{1}{3} \nabla(\nabla \cdot \mathbf{u})\right)
$$

in Cartesian coordinates for the velocity vector $\mathbf{u}=(u, v)^{\mathrm{T}}$. The present derivation may thus be more readily accessible than the one presented in Part I for the full Navier-Stokes equations under curvilinear coordinates. Like the BDF-ADI NavierStokes algorithms presented in Part I, the schemes discussed in this section incorporate three main elements, namely, (1) A BDF-based time discretization; (2) high-order extrapolation of relevant factors in quasilinear terms (the full compressible NavierStokes solver presented in Part I utilizes a similar procedure for nonquasilinear terms); and (3) the Douglas-Gunn ADI splitting.

The semi-discrete BDF scheme of order $s$ for equation (1) is given by

$$
\mathbf{u}^{n+1}=\sum_{j=0}^{s-1} a_{j} \mathbf{u}^{n-j}+b \Delta t\left(-\mathbf{u}^{n+1} \cdot \nabla \mathbf{u}^{n+1}+\mu\left(\Delta \mathbf{u}^{n+1}+\frac{1}{3} \nabla\left(\nabla \cdot \mathbf{u}^{n+1}\right)\right)\right)
$$

where $a_{j}$ and $b$ are the order-s BDF coefficients (see, e.g., [12, Chap. 3.12] or Part I); the truncation error associated with this scheme is a quantity of order $\mathcal{O}\left((\Delta t)^{s+1}\right)$. This equation is quasilinear: the derivatives of the solution appear linearly in the equation. Of course, the full compressible Navier-Stokes equations contain several nonquasilinear nonlinear terms. As detailed in Part I, by introducing a certain "quasilinear-like" form of the equations, all such nonlinear terms can be treated by an approach similar to the one described in this section. In preparation for a forthcoming ADI splitting we consider the somewhat more detailed form

$$
\begin{aligned}
\mathbf{u}^{n+1}=\sum_{j=0}^{s-1} a_{j} \mathbf{u}^{n-j}+b \Delta t( & -u^{n+1} \partial_{x}-v^{n+1} \partial_{y}+\mu\left(\begin{array}{cc}
4 / 3 & 0 \\
0 & 1
\end{array}\right) \partial_{x}^{2} \\
& \left.+\mu\left(\begin{array}{cc}
1 & 0 \\
0 & 4 / 3
\end{array}\right) \partial_{y}^{2}+\mu\left(\begin{array}{cc}
0 & 1 / 3 \\
1 / 3 & 0
\end{array}\right) \partial_{x} \partial_{y}\right) \mathbf{u}^{n+1}
\end{aligned}
$$

of equation (2), which we then rewrite as

$$
\begin{aligned}
& \left(I+b \Delta t\left(u^{n+1} \partial_{x}+v^{n+1} \partial_{y}-\mu\left(\begin{array}{cc}
4 / 3 & 0 \\
0 & 1
\end{array}\right) \partial_{x}^{2}-\mu\left(\begin{array}{cc}
1 & 0 \\
0 & 4 / 3
\end{array}\right) \partial_{y}^{2}\right)\right) \mathbf{u}^{n+1} \\
& \quad=\sum_{j=0}^{s-1} a_{j} \mathbf{u}^{n-j}+b \Delta t \mu\left(\begin{array}{cc}
0 & 1 / 3 \\
1 / 3 & 0
\end{array}\right) \partial_{x} \partial_{y} \mathbf{u}^{n+1} .
\end{aligned}
$$

Upon spatial discretization, the solution of equation (4) for the unknown velocity field $\mathbf{u}^{n+1}$ amounts to inversion of a (generally large) nonlinear system of equations. In order to avoid inversion of such nonlinear systems we rely on high-order 
extrapolation of certain nondifferentiated terms. This procedure eliminates the nonlinearities present in the equation while preserving the order of temporal accuracy of the algorithm. In detail, let $P_{u}$ (resp. $P_{v}$ ) denote the polynomial of degree $s-1$ that passes through $\left(t^{n-j+1}, u^{n-j+1}\right)$ (resp. through $\left.\left(t^{n-j+1}, v^{n-j+1}\right)\right)$ for $1 \leq j \leq s$, and define $\widetilde{u}_{s}^{n+1}=P_{u}\left(t^{n+1}\right)\left(\right.$ resp. $\left.\widetilde{v}_{s}^{n+1}=P_{v}\left(t^{n+1}\right)\right)$ and $\widetilde{\mathbf{u}}_{s}^{n+1}=\left(\widetilde{u}_{s}^{n+1}, \widetilde{v}_{s}^{n+1}\right)$. Then, substituting $\widetilde{u}_{s}^{n+1}$ and $\widetilde{v}_{s}^{n+1}$ (resp. $\widetilde{\mathbf{u}}_{s}^{n+1}$ ) for the undifferentiated terms $u^{n+1}$ and $v^{n+1}$ (resp. for the mixed derivative term) in equation (4), the alternative variablecoefficient linear semidiscrete scheme

$$
\begin{aligned}
& {\left[I+b \Delta t\left(\widetilde{u}_{s}^{n+1} \partial_{x}+\widetilde{v}_{s}^{n+1} \partial_{y}-\mu\left(\begin{array}{cc}
4 / 3 & 0 \\
0 & 1
\end{array}\right) \partial_{x}^{2}-\mu\left(\begin{array}{cc}
1 & 0 \\
0 & 4 / 3
\end{array}\right) \partial_{y}^{2}\right)\right] \mathbf{u}^{n+1}} \\
& \quad=\sum_{j=0}^{s-1} a_{j} \mathbf{u}^{n-j}+b \Delta t \mu\left(\begin{array}{cc}
0 & 1 / 3 \\
1 / 3 & 0
\end{array}\right) \partial_{x} \partial_{y} \widetilde{\mathbf{u}}_{s}^{n+1}
\end{aligned}
$$

results. Clearly the truncation errors inherent in the linear scheme (5) are of the same order as those associated with the original nonlinear scheme (4).

Even though equation (5) is linear, solution of (a spatially discretized version of) this equation requires inversion of a generally exceedingly large linear system at each time step. To avoid this difficulty we resort to a strategy of ADI type [14] and, more explicitly, to the Douglas-Gunn splitting [6]. To derive the Douglas-Gunn splitting we reexpress equation (5) in the factored form

$$
\begin{aligned}
{[I+} & \left.b \Delta t\left(\widetilde{u}_{s}^{n+1} \partial_{x}-\mu\left(\begin{array}{cc}
4 / 3 & 0 \\
0 & 1
\end{array}\right) \partial_{x}^{2}\right)\right] \\
& \times\left[I+b \Delta t\left(\widetilde{v}_{s}^{n+1} \partial_{y}-\mu\left(\begin{array}{cc}
1 & 0 \\
0 & 4 / 3
\end{array}\right) \partial_{y}^{2}\right)\right] \mathbf{u}^{n+1} \\
& =\sum_{j=0}^{s-1} a_{j} \mathbf{u}^{n-j}+b \Delta t \mu\left(\begin{array}{cc}
0 & 1 / 3 \\
1 / 3 & 0
\end{array}\right) \partial_{x} \partial_{y} \widetilde{\mathbf{u}}_{s}^{n+1} \\
& +(b \Delta t)^{2}\left(\widetilde{u}_{s}^{n+1} \partial_{x}-\mu\left(\begin{array}{cc}
4 / 3 & 0 \\
0 & 1
\end{array}\right) \partial_{x}^{2}\right)\left(\widetilde{v}_{s}^{n+1} \partial_{y}-\mu\left(\begin{array}{cc}
1 & 0 \\
0 & 4 / 3
\end{array}\right) \partial_{y}^{2}\right) \widetilde{\mathbf{u}}_{s-1}^{n+1} .
\end{aligned}
$$

We specially mention the presence of terms on the right-hand side this equation which only depend on solution values at times $t^{n}, \ldots, t^{n-s+1}$, and which have been incorporated to obtain an equation that is equivalent to (5) up to order $\mathcal{O}\left((\Delta t)^{s+1}\right)$.

Remark 2.1. It is important to note that, although $\widetilde{\mathbf{u}}_{s-1}^{n+1}$ provides an approximation of $\mathbf{u}^{n+1}$ of order $(\Delta t)^{s-1}$, the overall accuracy order inherent in the right-hand side of equation (6) is $(\Delta t)^{s+1}$, as needed - in view of the $(\Delta t)^{2}$ prefactor that occurs in the expression that contains $\widetilde{\mathbf{u}}_{s-1}^{n+1}$. Even though the approximation $\widetilde{\mathbf{u}}_{s}^{n+1}$ could have been used while preserving the accuracy order, we have found that use of the lower-order extrapolation $\widetilde{\mathbf{u}}_{s-1}^{n+1}$ is necessary to ensure stability.

Equation (6) can be expressed in the split form

$$
\begin{aligned}
{[I+} & \left.b \Delta t\left(\widetilde{u}_{s}^{n+1} \partial_{x}-\mu\left(\begin{array}{cc}
4 / 3 & 0 \\
0 & 1
\end{array}\right) \partial_{x}^{2}\right)\right] \mathbf{u}^{*} \\
& =\sum_{j=0}^{s-1} a_{j} \mathbf{u}^{n-j}+b \Delta t \mu\left(\begin{array}{cc}
0 & 1 / 3 \\
1 / 3 & 0
\end{array}\right) \partial_{x} \partial_{y} \widetilde{\mathbf{u}}_{s}^{n+1} \\
& -b \Delta t\left(\widetilde{v}_{s}^{n+1} \partial_{y}-\mu\left(\begin{array}{cc}
1 & 0 \\
0 & 4 / 3
\end{array}\right) \partial_{y}^{2}\right) \widetilde{\mathbf{u}}_{s-1}^{n+1},
\end{aligned}
$$

Copyright $@$ by SIAM. Unauthorized reproduction of this article is prohibited. 


$$
\begin{aligned}
{[I+} & \left.b \Delta t\left(\widetilde{v}_{s}^{n+1} \partial_{y}-\mu\left(\begin{array}{cc}
1 & 0 \\
0 & 4 / 3
\end{array}\right) \partial_{y}^{2}\right)\right] \mathbf{u}^{n+1} \\
& =\sum_{j=0}^{s-1} a_{j} \mathbf{u}^{n-j}+b \Delta t \mu\left(\begin{array}{cc}
0 & 1 / 3 \\
1 / 3 & 0
\end{array}\right) \partial_{x} \partial_{y} \widetilde{\mathbf{u}}_{s}^{n+1} \\
& -b \Delta t\left(\widetilde{u}_{s}^{n+1} \partial_{x}-\mu\left(\begin{array}{cc}
4 / 3 & 0 \\
0 & 1
\end{array}\right) \partial_{x}^{2}\right) \mathbf{u}^{*}
\end{aligned}
$$

that could be used to evolve the solution from time $t^{n}$ to time $t^{n+1}$. We note that these split equations can also be expressed in the form

$$
\begin{aligned}
& {\left[I+b \Delta t\left(\widetilde{u}_{s}^{n+1} \partial_{x}-\mu\left(\begin{array}{cc}
4 / 3 & 0 \\
0 & 1
\end{array}\right) \partial_{x}^{2}\right)\right] \mathbf{u}^{*}} \\
& =\sum_{j=0}^{s-1} a_{j} \mathbf{u}^{n-j}+b \Delta t \mu\left(\begin{array}{cc}
0 & 1 / 3 \\
1 / 3 & 0
\end{array}\right) \partial_{x} \partial_{y} \widetilde{\mathbf{u}}_{s}^{n+1} \\
& \quad-b \Delta t\left(\widetilde{v}_{s}^{n+1} \partial_{y}-\mu\left(\begin{array}{cc}
1 & 0 \\
0 & 4 / 3
\end{array}\right) \partial_{y}^{2}\right) \widetilde{\mathbf{u}}_{s-1}^{n+1}, \\
& {\left[I+b \Delta t\left(\widetilde{v}_{s}^{n+1} \partial_{y}-\mu\left(\begin{array}{cc}
1 & 0 \\
0 & 4 / 3
\end{array}\right) \partial_{y}^{2}\right)\right] \mathbf{u}^{n+1}} \\
& \quad=\mathbf{u}^{*}+b \Delta t\left(\widetilde{v}_{s}^{n+1} \partial_{y}-\mu\left(\begin{array}{cc}
1 & 0 \\
0 & 4 / 3
\end{array}\right) \partial_{y}^{2}\right) \widetilde{\mathbf{u}}_{s-1}^{n+1},
\end{aligned}
$$

which is equivalent to (7) - as can be checked by subtracting equation (7) from (7). The splitting (8) does not contain the term involving a differential operator applied to $\mathbf{u}^{*}$ on the right-hand side of (7) and it contains, instead, two instances of a term involving a differential operator applied to $\widetilde{\mathbf{u}}_{s-1}^{n+1}$. This term needs to be computed only once for each full timestep and therefore (8) leads to a somewhat less expensive algorithm than (7).

3. Unconditional and quasi-unconditional stability. This section reviews relevant ideas concerning stability in ODE and PDE theory, and it introduces the new notion of quasi-unconditional stability.

The concept of stability has been expressed in a number of closely related forms. Here we follow the definition given in [15, Sec. 7.3] for multi-step methods for linear problems: Let $\bar{\phi}^{n}=\left(u^{n+q-1}, \ldots, u^{n}\right)^{\mathrm{T}}$ be the vector of functions $u^{n}$ approximating the true solution $u\left(t_{n}\right), t_{n}=n \Delta t$, of the problem $u_{t}=A u$. Let the numerical scheme be given by a relation of the form

$$
\bar{\phi}^{n+1}=\bar{C}(\Delta t) \bar{\phi}^{n}
$$

for some linear operator $\bar{C}(\Delta t)$. Following [15, Sec. 3.2] it is assumed that the spatial meshsize $h$ is given as a function of $\Delta t$, i.e., $h=g(\Delta t)$ for some "meshsize function" $g$ (or, more generally, $h_{\ell}=g_{\ell}(\Delta t)$ for given meshsize function $g_{\ell}, \ell=1, \ldots, d$ ). The meshsize functions $g_{\ell}$ are assumed to yield compatible spatial discretizations - so that, for example, for a unit-square domain the functions $g_{\ell}$ take values in the set $\{h=1 / N: N$ is a positive integer $\}$.

Definition 3.1. The method (9) is (Lax-Richtmyer) stable up to time $T$ and under the meshsize function $g$ if for some constant $\tau>0$ the operators $\bar{C}(\Delta t)^{n}$ 
are uniformly bounded for all integers $n$, all discretizations $(h, \Delta t)$ satisfying $h=$ $g(\Delta t), 0<\Delta t<\tau$, and $0 \leq n \Delta t \leq T$. A time-stepping algorithm is said to be unconditionally (Lax-Richtmyer) stable if, for some $\tau>0$, an upper bound exists for the operators $\bar{C}(\Delta t)^{n}$ which does not depend on either the meshsize function $g$ or the timestep value $\Delta t(0<\Delta t<\tau)$, provided $0 \leq n \Delta t \leq T$.

According to the equivalence theorem [15, Sec. 7.3], for a properly posed initialvalue problem and a consistent multistep discrete approximation, stability is necessary and sufficient for convergence.

Equivalently, the concepts of stability and unconditional stability can be expressed in terms of domains in discretization space $(h, \Delta t)$. Conditional stability then occurs provided the uniform boundedness of the operators $\bar{C}(h, \Delta t)^{n}$ takes place for all $(h, \Delta t)$ in a certain region of discretization parameter space (whose closure contains the origin) restricted by (intersected with) the set of $\Delta t$ satisfying the condition $0 \leq n \Delta t \leq T$. A method is unconditionally stable if $\bar{C}^{n}$ is uniformly bounded for all positive pairs $(h, \Delta t)$ provided $0 \leq n \Delta t \leq T$.

The region of absolute stability $R$ of an ODE scheme, in turn, is the set of complex numbers $z=\lambda \Delta t$ for which the numerical solution of the ODE $y^{\prime}(t)=\lambda y(t)$ is stable for the timestep $\Delta t$. A numerical method which is stable for all $\Delta t>0$ and for all $\lambda$ with negative real part is said to be $A$-stable. In fact, the first- and second-order BDF ODE solvers are A-stable, and thus may lead to unconditionally stable methods for certain types of linear PDEs. As is well known, however, implicit linear multistep methods of order greater than 2, and, in particular, the BDF schemes of order $s \geq 3$, are not A-stable (Dahlquist's second barrier [5]). Nevertheless, we will see that PDE solvers based on such higher-order BDF methods may enjoy the property of quasiunconditional stability - a concept that we define in what follows.

DEFINITION 3.2. Let $\Omega_{h}$ be a family of spatial discretizations of a domain $\Omega$ controlled by a mesh-size parameter $h$ and let $\Delta t$ be a temporal step size. A numerical method for the solution of the $P D E Q_{t}=\mathcal{P} Q$ in $\Omega$ is said to be quasiunconditionally stable if there exist positive constants $M_{h}$ and $M_{t}$ such that the method is stable for all $h<M_{h}$ and all $\Delta t<M_{t}$.

Clearly, quasi-unconditional stability implies that, for small enough $\Delta t$, the method is stable for arbitrarily fine spatial discretizations. Note that stability may still take place outside of the quasi-unconditional stability rectangle $\left(0, M_{h}\right) \times\left(0, M_{t}\right)$ provided additional stability constraints are satisfied. For example, Figure 2 presents a schematic of the stability region for a notional method that enjoys quasi-unconditional stability in the parameter space $(h, \Delta t)$ as well as conditional CFL-like stability outside the quasi-unconditional stability rectangle. In practice we have encountered quasi-unconditionally stable methods whose stability outside the window $\left(0, M_{h}\right) \times$ $\left(0, M_{t}\right)$ is delimited by an approximately straight curve similar to that displayed in Figure 2 .

In lieu of a full stability analysis for the main problem under consideration (the fully nonlinear compressible Navier-Stokes equations, for which stability analyses are not available for any of the various extant algorithms), in support of the stability behavior observed in our numerical experiments we present rigorous stability results for simpler related problems. In particular, section 4 establishes a weak form of unconditional stability of the Fourier-based BDF2-ADI scheme for linear constant coefficient hyperbolic and parabolic equations in two spatial dimensions. Section 5, in turn, shows that quasi-unconditional stability takes place for Fourier-spectral BDF methods of order $s(2 \leq s \leq 6$, without ADI) for the advection-diffusion equation in one- 


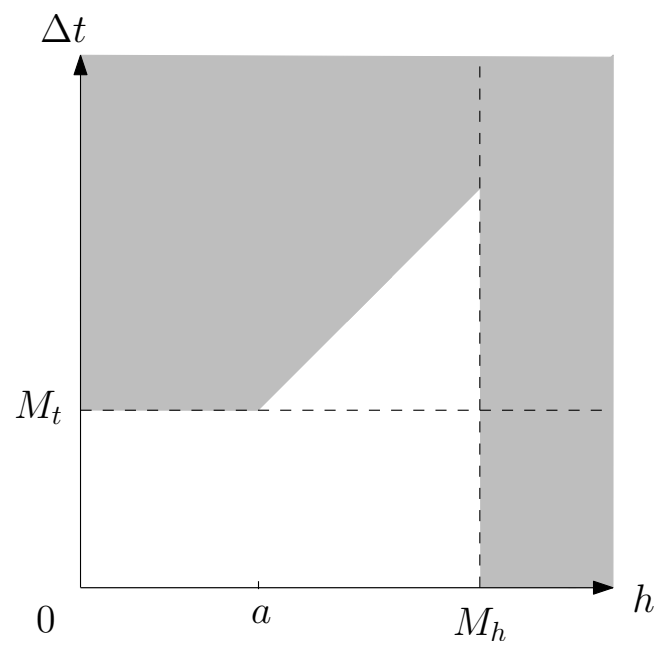

FIG. 2. Stability region of a notional quasi-unconditionally stable method. The white region is the set of $(h, \Delta t)$ pairs for which the method is stable. Note that, outside of the quasi-unconditional stability rectangle $\left(0, M_{h}\right) \times\left(0, M_{t}\right)$, the present hypothetical method is stable for timesteps satisfying the conditions $\Delta t<\min \left\{h, M_{h}\right\}$; other types of CFL-like conditions do, of course, occur commonly in practice. Thus, in particular, quasi-unconditional stability does not exclude the possibility of stability outside the rectangle $\left(0, M_{h}\right) \times\left(0, M_{t}\right)$.

and two-dimensional space, and section 6 presents numerical tests that demonstrate quasi-unconditional stability for the full compressible Navier-Stokes equations.

4. Stability of BDF2-ADI: Periodic linear case. This section presents stability results for BDF-ADI algorithms of order 2. In particular, these results (whose proofs are presented in Appendices A and B) establish energy bounds for BDF2-ADI schemes for the constant coefficient hyperbolic and parabolic equations with periodic boundary conditions under a Fourier collocation spatial approximation (sections 4.3 and 4.4). The energy bounds lead to a weak form of unconditional stability, as expressed in Corollaries 4.2 and 4.4. In the case of the parabolic equation, furthermore, a closely related result, presented in section 4.5 , establishes the corresponding stability of BDF-ADI-based Legendre polynomial spectral collocation method with (nonperiodic) homogeneous boundary conditions. Unfortunately, as discussed in that section, such a direct extension to the nonperiodic case has not been obtained for the hyperbolic equation.

4.1. Preliminary definitions. We consider the domain

$$
\Omega=[0,2 \pi) \times[0,2 \pi),
$$

which we discretize on the basis of an odd number $N+1$ of discretization points $(N$ even, for definiteness) in both the $x$ and $y$ directions: letting $h=2 \pi /(N+1), x_{j}=j h$ and $y_{k}=k h(0 \leq j, k \leq N)$, we use the two-dimensional grid

$$
\left\{\left(x_{j}, y_{k}\right): 0 \leq j, k \leq N\right\}
$$

(The restriction to even values of $N$, which is introduced for notational simplicity, allows us to avoid changes in the form of the summation limits in the Fourier series (14). Similarly, our use of equal numbers of points in the $x$ and $y$ directions simplifies the 
presentation somewhat. But, clearly, extensions of our constructions that allow for odd values of $N$ as well as unequal numbers of points in the $x$ and $y$ direction are straightforward.)

For (complex valued) grid functions

$$
f=\left\{f_{j k}\right\} \text { and } g=\left\{g_{j k}\right\}, \quad 0 \leq j, k \leq N
$$

we define the discrete inner product and norm

$$
\begin{gathered}
(f, g)=\frac{1}{(N+1)^{2}} \sum_{j, k} f_{j k} \bar{g}_{j k}, \\
|f|=\sqrt{(f, f)} .
\end{gathered}
$$

Each grid function $f$ as in (12) can be associated to a trigonometric interpolant $f_{N}(x, y)\left(f_{N}\left(x_{j}, y_{k}\right)=f_{j k}\right)$ which is given by

$$
f_{N}(x, y)=\sum_{|j|,|k| \leq \frac{N}{2}} \widehat{f}_{j k} e^{i(j x+k y)},
$$

where

$$
\widehat{f}_{j k}=\frac{1}{(N+1)^{2}} \sum_{\ell, m} f_{\ell m} e^{-i\left(j x_{\ell}+k y_{m}\right)} .
$$

Note that the inner product (13) coincides with the trapezoidal quadrature rule applied to the grid functions $f$ and $g$ over the underlying domain $[0,2 \pi) \times[0,2 \pi)$. Since the trapezoidal rule is exact for all truncated Fourier series containing exponentials of the form $e^{-i(j x+k y)}$ with $-N \leq j, k \leq N$, it follows that the discrete inner product (13) equals the integral inner product of the corresponding trigonometric interpolants -i.e.,

$$
(f, g)=\frac{1}{(2 \pi)^{2}} \int_{0}^{2 \pi} \int_{0}^{2 \pi} f_{N}(x, y) \bar{g}_{N}(x, y) d x d y
$$

In order to discretize solutions of PDEs we utilize time sequences of grid functions $u=\left\{u^{n}: n \geq 0\right\}$, where, for each $n, u^{n}=\left\{u_{j k}^{n}\right\}$ is a grid function such as those displayed in equation (12). For such time series the scalar product (13) at fixed $n$ can be used to produce a time series of scalar products. The inner product of two time series of grid functions $u=\left\{u^{n}: n \geq 0\right\}$ and $v=\left\{v^{n}: n \geq 0\right\}$ is thus a time series of complex numbers:

$$
(u, v)=\left\{\left(u^{n}, v^{n}\right): n \geq 0\right\} .
$$

4.2. Discrete spatial and temporal operators. In order to discretize PDEs we use discrete spatial and temporal differentiation operators that act on grid functions and time series, respectively.

We consider spatial differentiation first: the Fourier $x$-derivative operator $\delta_{x}$ applied to a grid function $f$, for example, is defined as the grid function $\delta_{x} f$ whose $j k$ value equals the value of the derivative of the interpolant $f_{N}$ at the point $\left(x_{j}, y_{k}\right)$ :

$$
\left(\delta_{x} f\right)_{j k}=\frac{\partial}{\partial x} f_{N}\left(x_{j}, y_{k}\right)
$$

The operators $\delta_{x x}, \delta_{y}, \delta_{y y}, \delta_{x y}=\delta_{x} \delta_{y}=\delta_{y} \delta_{x}$, etc. are defined similarly. 
Using the exactness relation (15) and integration by parts together with the periodicity of the domain, it follows that the first derivative operators $\delta_{x}$ and $\delta_{y}$ are skew-Hermitian and the second derivative operators $\delta_{x x}, \delta_{y y}$ are Hermitian:

$\left(\delta_{x} f, g\right)=-\left(f, \delta_{x} g\right),\left(\delta_{y} f, g\right)=-\left(f, \delta_{y} g\right),\left(\delta_{x x} f, g\right)=\left(f, \delta_{x x} g\right), \quad\left(\delta_{y y} f, g\right)=\left(f, \delta_{y y} g\right)$.

Certain temporal differentiation and extrapolation operators we use, in turn, produce a new time series for a given time series - for both numerical time series as well as time series of grid functions. These operators include the regular first- and second-order finite difference operators $D$ and $D^{2}$, the three-point backward difference operator $\widehat{D}$ that is inherent in the BDF2 algorithm, as well as the second-order accurate extrapolation operator " $\sim$ ":

$$
\begin{aligned}
(D u)^{n} & =u^{n}-u^{n-1}, & & n \geq 1, \\
\left(D^{2} u\right)^{n} & =(D u)^{n}-(D u)^{n-1}=u^{n}-2 u^{n-1}+u^{n-2}, & & n \geq 2, \\
(\widehat{D} u)^{n} & =\frac{3}{2} u^{n}-2 u^{n-1}+\frac{1}{2} u^{n-2}, & & n \geq 2, \\
\widetilde{u}^{n+1} & =2 u^{n}-u^{n-1}, & & n \geq 1 .
\end{aligned}
$$

Note that the members of the time series $\widehat{D} u$ can also be expressed in the forms

$$
\begin{aligned}
(\widehat{D} u)^{n} & =D\left(u^{n}+\frac{1}{2}(D u)^{n}\right) \\
& =\frac{1}{2}\left((D u)^{n}+(D \widetilde{u})^{n+1}\right) \\
& =\frac{3}{2}(D u)^{n}-\frac{1}{2}(D u)^{n-1} .
\end{aligned}
$$

In what follows we make frequent use of the finite difference product rule for two time series $u$ and $v$ :

$$
u D v=D(u v)-v D u+(D u)(D v) .
$$

An immediate consequence of (25), which will also prove useful, concerns the real part of scalar products of the form $(D u, P u)$ where $P$ is an operator which is self-adjoint with respect to the discrete inner product (13) and which commutes with $D$. For such operators we have the identity

$$
\Re(D u, P u)=\frac{1}{2} D(u, P u)+\frac{1}{2}(D u, P D u)
$$

which follows easily from the relations

$$
\begin{aligned}
(D u, P u) & =D(u, P u)-(u, D P u)+(D u, D P u) \\
& =D(u, P u)-(P u, D u)+(D u, P D u) \\
& =D(u, P u)-\overline{(D u, P u)}+(D u, P D u) .
\end{aligned}
$$

4.3. Periodic BDF2-ADI stability: Hyperbolic equation. This section establishes a weak form of unconditional stability of the BDF2-ADI method for the constant-coefficient advection equation

$$
U_{t}+\alpha U_{x}+\beta U_{y}=0
$$

Copyright (c) by SIAM. Unauthorized reproduction of this article is prohibited. 
in the domain (10), with real constants $\alpha$ and $\beta$, and subject to periodic boundary conditions. The BDF2-ADI scheme for the advection equation can be obtained easily by adapting the corresponding form (6) of the BDF2-ADI scheme for the pressure-free momentum equation. Indeed, using the Fourier collocation approximation described in the previous two sections, letting $u$ denote the discrete approximation of the solution $U$, and noting that, in the present context the necessary extrapolated term $\widetilde{\mathbf{u}}_{s-1}^{n+1}$ in equation (6) equals $u^{n}$, the factored form of our BDF2-ADI algorithm for equation (27) is given by

$$
\left(I+b \Delta t \alpha \delta_{x}\right)\left(I+b \Delta t \beta \delta_{y}\right) u^{n+1}=a_{0} u^{n}+a_{1} u^{n-1}+\alpha \beta(b \Delta t)^{2} \delta_{x} \delta_{y} u^{n} .
$$

Before proceeding to our stability result we derive a more convenient (equivalent) form for equation (28): using the numerical values $a_{0}=4 / 3, a_{1}=-1 / 3$, and $b=2 / 3$ of the BDF2 coefficients (see, e.g., [12, Chap. 3.12] or Part I), the manipulations

$$
\begin{aligned}
0 & =\left(I+b \Delta t \alpha \delta_{x}\right)\left(I+b \Delta t \beta \delta_{y}\right) u^{n+1}-a_{0} u^{n}-a_{1} u^{n-1}-\alpha \beta(b \Delta t)^{2} \delta_{x} \delta_{y} u^{n} \\
& =u^{n+1}-a_{0} u^{n}-a_{1} u^{n-1}+b \Delta t \alpha \delta_{x} u^{n+1}+b \Delta t \beta \delta_{y} u^{n+1}+\alpha \beta(b \Delta t)^{2} \delta_{x} \delta_{y}\left(u^{n+1}-u^{n}\right) \\
& =\frac{1}{b}\left(u^{n+1}-a_{0} u^{n}-a_{1} u^{n-1}\right)+\Delta t \alpha \delta_{x} u^{n+1}+\Delta t \beta \delta_{y} u^{n+1}+b \alpha \beta(\Delta t)^{2} \delta_{x} \delta_{y}\left(u^{n+1}-u^{n}\right)
\end{aligned}
$$

reduce equation (28) to the form

$$
\widehat{D} u+A u+B u+b A B D u=0,
$$

where $b=2 / 3, A=\alpha \Delta t \delta_{x}$, and $B=\beta \Delta t \delta_{y}$.

We are now ready to present an energy stability estimate for the BDF2-ADI equation (28).

THEOREM 4.1. The solution $u$ of (28) with initial conditions $u^{0}$ and $u^{1}$ satisfies

$$
\left|u^{n}\right|^{2}+\left|\widetilde{u}^{n+1}\right|^{2}+\frac{2}{3}\left(\left|A u^{n}\right|^{2}+\left|B u^{n}\right|^{2}+\sum_{m=2}^{n}\left|\left(D^{2} u\right)^{m}\right|^{2}\right) \leq M
$$

for all $n \geq 2$, where

$$
M=\left|u^{1}\right|^{2}+\left|\widetilde{u}^{2}\right|^{2}+\frac{2}{3}\left(\left|A u^{1}\right|^{2}+\left|B u^{1}\right|^{2}\right) .
$$

Proof. See Appendix A.

The following Corollary relates the previous result to the stability concepts discussed in section 3, and it implies, in particular, that the scheme (28) is Lax-Richtmyer stable (and therefore convergent [15, Sec. 7.3]) under a CFL relation of the form $\Delta t \leq C h$ for arbitrarily large values of $C$. To that end, following [15], for a given constant $c$ we define the compatible meshsize function

$$
g(\Delta t)=\llbracket c \Delta t \rrbracket,
$$

where the notation $\llbracket c \Delta t \rrbracket$ is defined in terms of the "integer-part" function $\lfloor\cdot\rfloor$ by means of the relation

$$
\llbracket a \rrbracket=2 \pi\left\lfloor\frac{2 \pi}{a}\right\rfloor^{-1} .
$$

(Note that the constant $c$ corresponds, roughly, to $C^{-1}$.) With these definitions it follows that $h=g(\Delta t)$ is compatible with the uniform discretization of the $2 \pi$ sized domain for all $\Delta t>0(g(\Delta t)=2 \pi /(N+1)$ for some integer $N)$. 
COROLlary 4.2. For each fixed value of the spatial discretization parameter $h$ the temporal scheme (28) is unconditionally stable: for any given $\tau>0$ there exists a constant $K$ (which may depend on $h$ and $\tau$ ) such that for any value of $\Delta t(0<\Delta t<\tau)$ we have $\left|u^{n}\right| \leq K$ for all $n \geq 2$. Further, for any final time $T>0$ the scheme is Lax-Richtmyer stable according to Definition 3.1 with meshsize function (30) and with arbitrarily small proportionality constant $c>0$.

Proof. In view of equation (9) with $\bar{\phi}^{n}=\left(u^{n+1}, u^{n}\right)^{\mathrm{T}}$ it suffices to show that $u^{n}$ is a bounded sequence under the norm $|\cdot|$. The result follows from the uniform boundedness, under the assumed prescription (30), of the terms $\left|A u^{1}\right|^{2}$ and $\left|B u^{1}\right|^{2}$ on the right-hand side of the energy bound given in Theorem 4.1. But the necessary uniform boundedness is established easily: calling $N=2 \pi / g(\Delta t)-1$ and noting that $(N+1)=2 \pi / g(\Delta t)$ and $g(\Delta t) \geq c \Delta t$, we have

$$
\begin{aligned}
\left|A u^{1}\right|^{2} & =\alpha^{2} \Delta t^{2}\left|\delta_{x} u^{1}\right|^{2} \\
& \leq \alpha^{2} \Delta t^{2}\left(\frac{N}{2}\right)^{2}\left|u^{1}\right|^{2} \\
& \leq \alpha^{2} \frac{\pi^{2}}{c^{2}}\left|u^{1}\right|^{2}
\end{aligned}
$$

and similarly for $\left|B u^{1}\right|^{2}$.

4.4. Fourier-based BDF2-ADI stability: Parabolic equation. The stability theory of the previous section can be extended to the parabolic case. Indeed, the present section establishes a weak form of unconditional stability for the BDF2-ADI method for the constant-coefficient parabolic equation

$$
U_{t}=\alpha U_{x x}+\beta U_{y y}+\gamma U_{x y} .
$$

Note the inclusion of the mixed derivative term, which is treated explicitly using temporal extrapolation in the BDF-ADI algorithm. Theorem 4.3 in this section proves, in particular, that extrapolation of the mixed derivative does not compromise the stability of the method.

The parabolicity conditions $\alpha>0, \beta>0$, and

$$
\gamma^{2} \leq 4 \alpha \beta
$$

which are assumed throughout this section, ensure that

$$
\int_{0}^{2 \pi} \int_{0}^{2 \pi} f\left(\alpha f_{x x}+\beta f_{y y}+\gamma f_{x y}\right) d x d y \leq 0
$$

for any twice continuously differentiable biperiodic function $f$ defined in the domain (10) - as can be established easily by integration by parts and completion of the square in the sum $\alpha\left(f_{x}\right)^{2}+\gamma f_{x} f_{y}$ together with some simple manipulations. In preparation for the parabolic-equation stability proof, in what follows we present a few preliminaries concerning the BDF2-ADI algorithm for equation (32).

We first note that a calculation similar to that leading to equation (29) shows that the Fourier-based BDF2-ADI scheme for (32) can be expressed in the form

$$
\widehat{D} u-\Delta t\left(\alpha \delta_{x x}+\beta \delta_{y y} u+\gamma \delta_{x} \delta_{y}\right) u+\Delta t \gamma \delta_{x} \delta_{y} D^{2} u+b(\Delta t)^{2} \alpha \beta \delta_{x x} \delta_{y y} D u=0
$$


Letting

$$
\begin{aligned}
& A=-\Delta t \alpha \delta_{x x}, \\
& B=-\Delta t \beta \delta_{y y}, \\
& F=-\Delta t \gamma \delta_{x} \delta_{y}, \\
& L=A+B+F,
\end{aligned}
$$

equation (35) becomes

$$
\widehat{D} u+L u-F D^{2} u+b A B D u=0 .
$$

Note that the operators $A$ and $B$ above do not coincide with the corresponding $A$ and $B$ operators in section 4.3 .

In view of both, the exactness relation (15) and the Fourier differentiation operators used (cf. (16)), it follows that $A, B, A B$, and $L$ are positive semidefinite operators. Indeed, in view of equation (34), for example, we have

$$
(u, L u)=-\frac{\Delta t}{(2 \pi)^{2}} \int_{0}^{2 \pi} \int_{0}^{2 \pi} u_{N}\left(\alpha\left(u_{N}\right)_{x x}+\beta\left(u_{N}\right)_{y y}+\gamma\left(u_{N}\right)_{x y}\right) d x d y \geq 0
$$

similar relations for $A, B$, and $A B$ follow directly by integration by parts.

Finally we present yet another consequence of the parabolicity condition (33) which will prove useful: for any grid function $g$ we have

$$
|F g|^{2}=\gamma^{2}(\Delta t)^{2}\left(\delta_{x} \delta_{y} g, \delta_{x} \delta_{y} g\right) \leq 4 \alpha \beta(\Delta t)^{2}\left(g, \delta_{x}^{2} \delta_{y}^{2} g\right)=4(g, A B g) .
$$

Thus, defining the seminorm

$$
|u|_{P}=\sqrt{(u, P u)}
$$

for a given positive semidefinite operator $P$ and using $P=A B$ we obtain

$$
|F g|^{2} \leq 4|g|_{A B}^{2}
$$

The following theorem can now be established.

TheOREM 4.3. The solution $u$ of the Fourier-based BDF2-ADI scheme (35) for equation (32) with initial conditions $u^{0}, u^{1}$ satisfies

$$
\frac{1}{4}\left|u^{n}\right|^{2}+\frac{1}{4}\left|\widetilde{u}^{n+1}\right|^{2}+\frac{1}{3}\left|(D u)^{n}\right|_{A B}^{2}+\frac{1}{4} \sum_{m=1}^{n}\left|D^{2} u\right|^{2}+\sum_{m=1}^{n}\left|u^{n}\right|_{L}^{2} \leq M
$$

for $n \geq 2$, where

$$
\begin{aligned}
M= & \frac{1}{4}\left|u^{1}\right|^{2}+\frac{1}{4}\left|\widetilde{u}^{2}\right|^{2}+\frac{1}{3}\left|u^{1}\right|_{A B}^{2}+3\left|u^{1}\right|_{L}-\Re\left(u^{1}, F(D u)^{1}\right) \\
& +3\left|(D u)^{1}\right|^{2}+\frac{3}{2}\left(\left|(D u)^{1}\right|_{A}^{2}+\left|(D u)^{1}\right|_{B}^{2}\right)+\frac{1}{3}\left|(D u)^{1}\right|_{A B}^{2} .
\end{aligned}
$$

Proof. See Appendix B.

We now establish a stability result analagous to Corollary 4.2 . We show that the scheme (35) is Lax-Richtmyer stable under a CFL relation of the form $\Delta t \leq C h^{2}$ for arbitrarily large values of $C$. The compatible meshsize function in this case is

$$
g(\Delta t)=\llbracket c \sqrt{\Delta t} \rrbracket,
$$

where $c$ is an arbitrary positive constant and the bracket (defined in equation (31)) ensures that $g(\Delta t)=2 \pi /(N+1)$ for some integer $N$.

Copyright (c) by SIAM. Unauthorized reproduction of this article is prohibited. 
Corollary 4.4. For each fixed value of the spatial discretization parameter $h$, the temporal scheme (35) is unconditionally stable: for any given $\tau>0$ there exists a constant $K$ (which may depend on $h$ and $\tau$ ) such that for any value of $\Delta t(0<\Delta t<\tau)$ we have $\left|u^{n}\right| \leq K$ for all $n \geq 2$. Further, for any final time $T>0$ the scheme is Lax-Richtmyer stable according to Definition 3.1 with meshsize function (43) and with arbitrarily small proportionality constant $c>0$.

Proof. It suffices to establish that, for all $\Delta t$ and $h=2 \pi /(N+1)$ satisfying $h=g(\Delta t)$ with $g$ given by (43), all the terms involving derivatives on the right-hand side of the energy bound in Theorem 4.3 are uniformly bounded as $\Delta t \rightarrow 0$. This is easily established from the fact that, up to constant factors, the operators $A, B, F$, and $L$ equal products of second-order differential operators multiplied by $\Delta t$. Taking the term $\left|(D u)^{1}\right|_{A}^{2}$ and relying on the fact that $\delta_{x}$ is skew-Hermitian, for example, we obtain

$$
\begin{aligned}
\left|(D u)^{1}\right|_{A}^{2} & =-\alpha \Delta t\left(u^{1}-u^{0}, \delta_{x}^{2}\left(u^{1}-u^{0}\right)\right) \\
& =\alpha \Delta t\left|\delta_{x}\left(u^{1}-u^{0}\right)\right|^{2} \\
& \leq \alpha \Delta t\left(\frac{N}{2}\right)^{2}\left(\left|u^{1}\right|^{2}+\left|u^{0}\right|^{2}\right) \\
& \leq \frac{\alpha \pi^{2}}{c^{2}}\left(\left|u^{1}\right|^{2}+\left|u^{0}\right|^{2}\right) .
\end{aligned}
$$

and similarly for the other terms.

4.5. Nonperiodic (Legendre based) BDF2-ADI stability: Parabolic equation. The stability result provided in the previous section for the parabolic equation with periodic boundary conditions can easily be extended to a nonperiodic context using Legendre expansions; the present section outlines the corresponding stability proof. Background on the polynomial collocation methods may be found, e.g., in [11].

Under Legendre collocation we discretize the domain $\Omega=[-1,1] \times[-1,1]$ by means of the $N+1$ Legendre Gauss-Lobatto quadrature nodes $x_{j}=y_{j}(j=0, \ldots, N)$ in each one of the coordinate directions, which defines the grid $\left\{\left(x_{j}, y_{k}\right): 0 \leq j, k \leq\right.$ $N\}$ ( with $x_{0}=y_{0}=-1$ and $x_{N}=y_{N}=1$ ). For real-valued grid functions $f=\left\{f_{j k}\right\}$ and $g=\left\{g_{j k}\right\}$ we use the inner product

$$
(f, g)=\sum_{j=0}^{N} \sum_{k=0}^{N} w_{j} w_{k} f_{j k} g_{j k},
$$

where $w_{\ell}(0 \leq \ell \leq N)$ are the Legendre Gauss-Lobatto quadrature weights. The interpolant $f_{N}$ of a grid function $f$ is a linear combination of the form

$$
f_{N}(x, y)=\sum_{j=0}^{N} \sum_{k=0}^{N} \widehat{f}_{j k} P_{j}(x) P_{k}(y)
$$

of Legendre polynomials $P_{j}$, where $\widehat{f}_{j k}$ are the Legendre coefficients of $f_{N}$.

Certain exactness relations related to the one we used in the Fourier case exist in the Legendre context as well. Namely, for grid functions $f$ and $g$ for which the product of the interpolants has polynomial degree $\leq 2 N-1$ in the $x$ (resp. y) variable, the $j$ (resp. $k$ ) summation in the inner product (44) of the two grid functions is equal 
to the integral of the product of their corresponding polynomial interpolants with respect to $x$ (resp. $y$ ) [9, Sec. 5.2.1], i.e.,

$$
\begin{gathered}
(f, g)=\sum_{k=0}^{N} \int_{-1}^{1} f_{N}\left(x, y_{k}\right) g_{N}\left(x, y_{k}\right) d x, \\
\text { provided } \\
\operatorname{deg}\left(f_{N}\left(x, y_{k}\right) g_{N}\left(x, y_{k}\right)\right) \leq 2 N-1 \text { for all } 0 \leq k \leq N, \\
\text { and } \\
(f, g)=\sum_{j=0}^{N} \int_{-1}^{1} f_{N}\left(x_{j}, y\right) g_{N}\left(x_{j}, y\right) d y, \\
\text { provided } \\
\operatorname{deg}\left(f_{N}\left(x_{j}, y\right) g_{N}\left(x_{j}, y\right)\right) \leq 2 N-1 \text { for all } 0 \leq j \leq N,
\end{gathered}
$$

Thus, for example, defining the Legendre $x$-derivative operator $\delta_{x}$ as the array of grid values of the derivative of the Legendre interpolant $f_{N}=f_{N}(x, y)$ (cf. (16)), with corresponding definitions for $\delta_{y}, \delta_{x x}, \delta_{y y}$, and $\delta_{x y}$, the exactness relation (46a) holds whenever one or both of the grid functions $f$ and $g$ is a Legendre $x$ derivative of a grid function $h=\left\{h_{j k}\right\}, 0 \leq j, k \leq N$.

As discussed in what follows, these properties of the Legendre polynomials lead to a stability proof for the nonperiodic parabolic problem

$$
\text { (47) } U_{t}=\alpha U_{x x}+\beta U_{y y}+\gamma U_{x y} \text { in } \Omega, \quad U=0 \text { on } \Gamma_{\text {Dir }}, \quad \text { and } \quad \frac{\partial U}{\partial n}=0 \text { on } \Gamma_{\mathrm{Neu}} \text {, }
$$

which enforces boundary conditions of Dirichlet and Neumann types on respective (disjoint) portions $\Gamma_{\text {Dir }}$ and $\Gamma_{\text {Neu }}$ of the domain boundary. This proof in fact results from a slight modification of the strategy presented for the periodic case in section 4.4 and Appendix B. Indeed, we note that the latter proof relies on the following properties of the spatial differentiation operators:

1. The discrete first and second derivative operators are skew-Hermitian and Hermitian, respectively.

2. The operators $A, B, L$, and $A B$ defined in section 4.4 are positive semidefinite.

Both of these results were established by exploiting (a) The exactness relation satisfied by the discrete and continuous inner products (section 4.1), as well as (b) The vanishing of boundary terms arising from integration by parts. As indicated above in this section, a variant of point (a) holds in the present context. Point (b) also holds, in view of the assumption of vanishing Dirichlet/Neumann boundary conditions in equation (47). We thus have the following theorem:

TheOREm 4.5. Let the scalar product $(\cdot, \cdot)$ and the differentiation operators $\delta_{x}$, etc., be defined as above in the present section 4.5. Then, the solution $u$ of the Legendre-based scheme of the form (35) for equation (47) with initial conditions $u^{0}$, $u^{1}$ satisfies the energy bound (41) for $n \geq 2$, where $M$ is given by equation (42).

The following corollary, which is a direct consequence of the energy bound in the previous theorem, extends the first part of Corollary 4.4 to the nonperiodic Legendre case.

COROLlany 4.6. For each fixed value of the spatial discretization parameter $h$ the temporal scheme (35) for the nonperiodic problem (47) is unconditionally stable:

Copyright (c) by SIAM. Unauthorized reproduction of this article is prohibited. 
that is, for any given $\tau>0$ there exists a constant $K$ (which may depend on $h$ and $\tau)$ such that for any value of $\Delta t(0<\Delta t<\tau)$ we have $\left|u^{n}\right| \leq K$ for all $n \geq 1$.

Remark 4.7. Unfortunately, we have not been able to extend the stability proof to the nonperiodic hyperbolic case. Indeed, since in this case only one boundary condition is specified in each spatial direction, not all boundary terms arising from integration by parts vanish - and, hence, the first derivative operators are generally not skew-Hermitian. See [8] for a discussion of stability proofs for spectral methods in the context of hyperbolic problems. Additionally, note that, unlike Corollaries 4.2 and 4.4, Corollary 4.6 does not contain a reference to Lax-Richtmyer stability. Such a result could be obtained by utilizing an optimal or otherwise reasonably sharp upper bound of the right-hand side in (42) in the Legendre case. The derivation of such a bound lies beyond the scope of this paper and the study of this problem is thus left for future work.

5. Quasi-unconditional stability for higher-order non-ADI BDF methods: Periodic advection-diffusion equation.

5.1. Rectangular window of stability. This paper does not present stability proofs for the BDF-ADI methods of order higher than 2. In order to provide some additional insights into the stability properties arising from the BDF strategy in the context of time-domain PDE solvers, this section investigates the stability of the BDF schemes of order $s \geq 2$ under periodic boundary conditions and Fourier discretizations. Because of Dahlquist's second barrier [12, p. 243] the $s \geq 3$ schemes cannot be unconditionally stable for general (even linear) PDEs. However, we will rigorously establish that the BDF methods of order $s$ with $2 \leq s \leq 6$ are quasi-unconditionally stable for the advection-diffusion equation, in the sense of Definition 3.2. (As shown in section 4 further, the $s=2$ algorithms are indeed unconditionally stable, at least for certain linear PDE.)

To introduce the main ideas in our quasi-unconditional stability analysis for BDFbased schemes we consider first a Fourier-BDF scheme for the advection-diffusion equation in one spatial dimension with periodic boundary conditions:

$$
\begin{aligned}
& U_{t}+\alpha U_{x}=\beta U_{x x}, \quad x \in \mathbb{R}, \quad t \geq 0, \\
& U(x, 0)=f(x), \quad U(x, t)=U(x+2 \pi, t),
\end{aligned}
$$

where $\beta>0$. Using the $N$-point Fourier discretization described in sections 4.1 and 4.2 , the resulting semidiscrete equation is given by

$$
\frac{\partial}{\partial t} u=\left(-\alpha \delta_{x}+\beta \delta_{x}^{2}\right) u .
$$

The von Neumann criterion provides a necessary and sufficient stability condition [13]: the order- $s$ scheme is stable if and only if the (complex!) eigenvalues of the spatial operator in the semidiscrete system (49) multiplied by $\Delta t$ lie within the region $R_{s}$ of absolute stability of the BDF method of order $s$. As is known [13, Sec. 7.6.1 and p. 174], further, the boundary of $R_{s}$, which will be denoted by $\partial R_{s}$, is given by the parametrization

$$
z(\theta)=x(\theta)+i y(\theta)=\frac{1}{b}\left(1-\sum_{j=0}^{s-1} a_{j} e^{-i(j+1) \theta}\right), \quad 0 \leq \theta \leq 2 \pi .
$$

Copyright $@$ by SIAM. Unauthorized reproduction of this article is prohibited. 


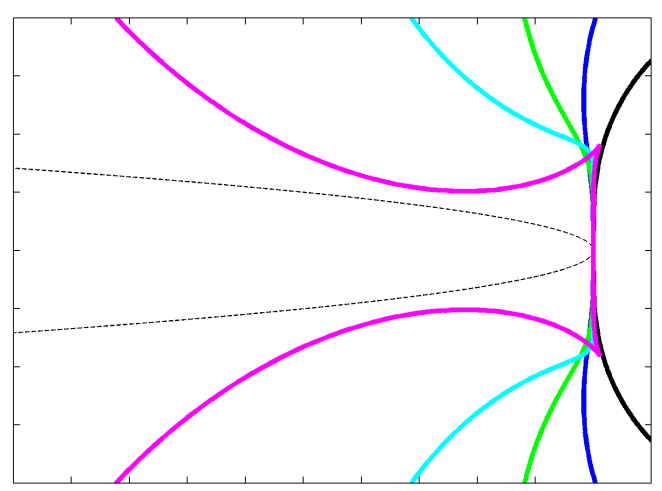

FIG. 3. Boundaries of the stability regions of the BDF methods of order $s=2$ (black), 3 (blue), 4 (green), 5 (cyan), and 6 (magenta), together with a parabola $\Gamma_{m}$ (dashed black), $m=0.1$, that is contained in every stability region.

We will see that, for the present one-dimensional advection-diffusion problem, the eigenvalues of the spatial operator lie on a parabola which does not vary with $N$. To establish the quasi-unconditional stability (Definition 3.2) of the Fourier-BDF scheme under consideration, it is therefore necessary and sufficient to show that a certain family of "complete parabolas" lie in the stability region of the BDF scheme for $\Delta t<M_{t}$ and $h<M_{h}$ for some constants $M_{t}$ and $M_{h}$ which define the corresponding rectangular window of stability. As discussed in the next section, furthermore, certain CFL-like stability constraints that hold outside of the rectangular window of stability are obtained by consideration of the relative position of eigenvalues on such parabolas and the BDF stability region. The former property (quasi-unconditional stability) follows from an application of Lemma 5.1, which establishes that the stability regions of the BDF schemes contain the required families of parabolas.

Lemma 5.1. Let $s$ denote an integer satisfying $2 \leq s \leq 6$ and let $m>0$ be a real constant. Further, let $\Gamma_{m}$ denote the locus of the left-facing parabola of equation $x=-\frac{1}{m} y^{2}$, let $\Gamma_{m}^{*}=\Gamma_{m} \backslash\{(0,0)\}$, and define

$$
m_{C}=m_{C}(s)=\inf \left\{m>0 \mid \Gamma_{m}^{*} \cap \partial R_{s} \neq \emptyset\right\},
$$

with the interpretation that $m_{C} \equiv+\infty$ when the set on the right-hand side is empty. Then $m_{C}>0$. In particular, for $2 \leq s \leq 6$ the stability region $R_{s}$ contains a family of nondegenerate left-facing parabolas (cf. Figure 3 ).

Proof. Since the $s$ th order BDF scheme $(2 \leq s \leq 6)$ is $\mathrm{A}(\gamma)$-stable for some $\gamma>0$ (see, e.g., [13, p. 175]), it suffices to show that, for some positive $m$ and $\varepsilon, R_{s}$ contains a parabolic region of the form $\left\{\left(x+i y:-\varepsilon<x<0\right.\right.$ and $\left.x<-\frac{1}{m} y^{2}\right\}$. But this follows directly from the asymptotics of the function $z(\theta)$ around $\theta=0$,

$$
\Re z(\theta) \sim C_{s} \cdot \theta^{2[s / 2+1]} \text { and } \Im z(\theta) \sim \theta,
$$

where $[t]$ denotes the largest integer that is less than or equal the real number $t$ and where $C_{s}$ is an $s$-dependent constant. These asymptotic relations, in turn, follow by substituting the Taylor expansion of $e^{-i(j+1) \theta}$ around $\theta=0$ in equation (50) and showing, by a simple direct calculation, that $\Im z(0)=0$, that $\left.(d \Im z / d \theta)\right|_{\theta=0}=1$, and that the lowest-order terms in the Taylor series of $\Re z$ around the origin vanish in accordance with equation (52). (Note that since the $s=2$ solver is A-stable we actually have $m_{C}=+\infty$ for $s=2$.) The proof is complete. 
TABLE 1

Approximate numerical values of the constant $m_{C}$ obtained via numerical evaluation of equation (51). For all $m<m_{C}$ the parabola $\Gamma_{m}$ described in Lemma 5.1 is contained in the region of absolute stability of the BDF method of order s. By Theorem 5.2, the order-s BDF method applied to the advection-diffusion equation $u_{t}+\alpha u_{x}=\beta u_{x x}$ with Fourier collocation is stable for all $\Delta t<\frac{\beta}{\alpha^{2}} m_{C}$.

\begin{tabular}{l|cccc}
\hline \hline$s$ & 3 & 4 & 5 & 6 \\
\hline$m_{C} \approx$ & 14.0 & 5.12 & 1.93 & 0.191 \\
\hline \hline
\end{tabular}

Numerical estimates of $m_{C}$ for each BDF method of orders 3 through 6 (which were computed as the infimum of $-\frac{y^{2}}{x}$ over the boundary of $R_{s}$ in the negative half of the complex plane), are presented in Table 1.

Theorem 5.2. Let $s$ and $N$ denote positive integers, $2 \leq s \leq 6$. Then, the sth order Fourier-based BDF scheme described in this section for the problem (48) is quasi-unconditionally stable (Definition 3.2) with $M_{h}=\infty$ and $M_{t}=\frac{\beta}{\alpha^{2}} m_{C}\left(M_{t}=\infty\right.$ for $\alpha=0)$, where the $s$-dependent constant $m_{C}$ is given in equation (51).

Proof. Applying the discrete Fourier transform,

$$
\widehat{u}_{k}=\frac{1}{N+1} \sum_{j=0}^{N} u_{j} e^{-i x_{j} k}, \quad-\frac{N}{2} \leq k \leq \frac{N}{2}
$$

to equation (49) we obtain the set of ODEs

$$
\frac{\partial}{\partial t} \widehat{u}_{k}=-\left(i \alpha k+\beta k^{2}\right) \widehat{u}_{k}
$$

for the Fourier coefficients $\widehat{u}_{k}$. It is clear from this transformed equation that the eigenvalues of the spatial operator for the semidiscrete system are given by

$$
\lambda(k)=-\left(i \alpha k+\beta k^{2}\right) .
$$

To complete the proof it suffices to show that these eigenvalues multiplied by $\Delta t$ lie in the stability region of the BDF method for all $\Delta t<\frac{\beta}{\alpha^{2}} m_{C}$.

Let $z_{k}=\lambda(k) \Delta t$. In the case $\alpha=0$ we have $z_{k} \leq 0$ for all integers $k$ and thus, since the BDF methods under consideration are $\mathrm{A}(\gamma)$-stable for some $\gamma>0[13$, p. 175], we immediately see that the Fourier-based BDF scheme described in the present section methods are unconditionally stable, and, thus, $M_{h}=\infty$ and $M_{t}=\infty$ in this case, as claimed. For the case $\alpha \neq 0$, in turn, we have

$$
\begin{aligned}
z_{k} & =\lambda(k) \Delta t=-\beta \Delta t k^{2}-i \alpha \Delta t k \\
& =-\frac{1}{\frac{\alpha^{2} \Delta t}{\beta}}(\alpha \Delta t k)^{2}-i(\alpha \Delta t k) \quad \text { with } \quad-\frac{N}{2} \leq k \leq \frac{N}{2} .
\end{aligned}
$$

From (55) it is clear that, for all integers $k, z_{k}$ lies on the left-facing parabola $\Gamma_{m}$ with

$$
m=\frac{\alpha^{2} \Delta t}{\beta} .
$$

But, by Lemma 5.1 we know that the parabola $\Gamma_{m}$ lies within the stability region $R_{s}$ for all $m<m_{C}$, and, thus, for all

$$
\Delta t<\frac{\beta}{\alpha^{2}} m_{C} .
$$

Copyright (c) by SIAM. Unauthorized reproduction of this article is prohibited. 
We have thus shown that, provided (57) holds, $z_{k}$ lies within $R_{s}$ for all $k$-and, therefore, in the case $\alpha \neq 0$, the algorithm is quasi-unconditionally stable with $M_{t}=\frac{\beta}{\alpha^{2}}$ and $M_{h}=\infty$ as well. The proof is thus complete.

We now establish the quasi-unconditional stability of the Fourier-based BDF methods for the advection-diffusion equation

$$
u_{t}+\boldsymbol{\alpha} \cdot \nabla u=\beta \Delta u \quad \text { in }[0,2 \pi]^{d}, d=2,3
$$

in two- and three-dimensional space and with periodic boundary conditions, where $\boldsymbol{\alpha}=\left(\alpha_{1}, \alpha_{2}\right)^{\mathrm{T}}$ and $\boldsymbol{\alpha}=\left(\alpha_{1}, \alpha_{2}, \alpha_{3}\right)^{\mathrm{T}}$ for $d=2$ and 3 respectively. Thus, letting $\mathbf{x}=(x, y)^{\mathrm{T}}, \mathbf{k}=\left(k_{x}, k_{y}\right)^{\mathrm{T}}, \mathbf{N}=\left(N_{x}, N_{y}\right)^{\mathrm{T}}\left(\right.$ resp. $\mathbf{x}=(x, y, z)^{\mathrm{T}}, \mathbf{k}=\left(k_{x}, k_{y}, k_{z}\right)^{\mathrm{T}}$, $\left.\mathbf{N}=\left(N_{x}, N_{y}, N_{z}\right)^{\mathrm{T}}\right)$ in $d=2$ (resp. $d=3$ ) spatial dimensions, and substituting the Fourier series (using multi-index notation)

$$
u(\mathbf{x})=\sum_{\mathbf{k}=-\mathbf{N} / 2}^{\mathbf{N} / 2} \widehat{u}_{\mathbf{k}} e^{i(\mathbf{k} \cdot \mathbf{x})}
$$

into equation (58), the Fourier-based BDF method of order $s$ results as the $s$-order BDF method applied to the ODE system

$$
\frac{\partial \widehat{u}_{\mathbf{k}}}{\partial t}=\left(-i(\boldsymbol{\alpha} \cdot \mathbf{k})-\beta|\mathbf{k}|^{2}\right) \widehat{u}_{\mathbf{k}}
$$

for the Fourier coefficients $\widehat{u}_{\mathbf{k}}$. (In order to utilize a single meshsize parameter $h$ and corresponding quasi-unconditional stability constant $M_{h}$ while allowing for different grid-fineness in the $x, y$, and $z$ directions, we utilize positive integers $r_{2}$ and $r_{3}$ and discretize the domain on the basis of $N_{x}+1$ points in the $x$ direction, $N_{y}+1=r_{2} N_{x}+1$ points in the $y$ direction, and $N_{z}+1=r_{3} N_{x}+1$ points in the $z$ direction ( $N_{x}$ even). The mesh size parameter is then given by $h=2 \pi /\left(N_{x}+1\right)$.)

TheOREm 5.3. The Fourier-based BDF scheme of order $s$ (not ADI!) for the problem (58) with $3 \leq s \leq 6$ is quasi-unconditionally stable with constants $M_{t}=$ $\frac{\beta}{|\boldsymbol{\alpha}|^{2}} m_{C}$ and $M_{h}=\infty$.

Proof. We first note that the eigenvalues of the discrete spatial operator in equation (59) multiplied by $\Delta t$ are given by

$$
z_{\mathbf{k}}=-i \Delta t \boldsymbol{\alpha} \cdot \mathbf{k}-\Delta t \beta|\mathbf{k}|^{2} .
$$

Clearly, in contrast with the situation encountered in the context of the one dimensional problem considered in Theorem 5.2, in the present case the set of $z_{\mathbf{k}}$ does not lie on a single parabola. But, to establish quasi-unconditional stability it suffices to verify that this set is bounded on the right by a certain left-facing parabola through the origin. This can be accomplished easily: in view of the Cauchy-Schwarz inequality, we have

$$
|\boldsymbol{\alpha} \cdot \mathbf{k}| \leq|\boldsymbol{\alpha}||\mathbf{k}|
$$

Therefore, letting $\xi=|\mathbf{k}|$, the eigenvalues multiplied by $\Delta t$ are confined to the region

$$
\left\{z: \Re z=-\Delta t \beta \xi^{2},|\Im z| \leq \Delta t|\boldsymbol{\alpha}| \xi, \xi \geq 0\right\} .
$$




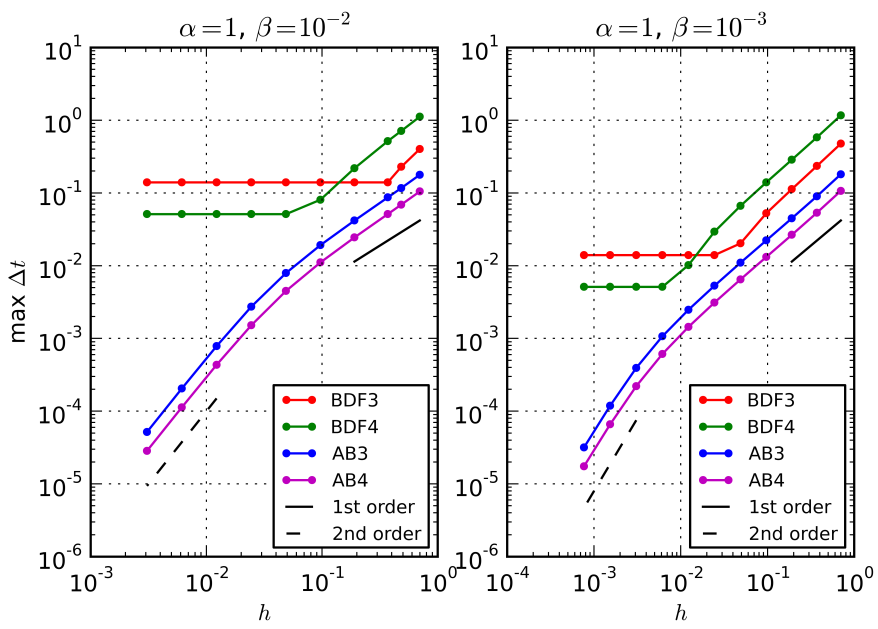

FIG. 5. Maximum stable $\Delta t$ versus spatial mesh size $h$ for Fourier-based BDF and AB methods of orders 3 and 4 for the advection-diffusion equation (48), with $\alpha=1, \beta=10^{-2}$ (left plot) and $\alpha=1, \beta=10^{-3}$ (right plot). Both the BDF and AB maximum-stable- $\Delta t$ values were obtained by considering the intersection of the boundary locus of the relevant stability region (either BDF or $A B)$, as indicated in section 5.2 in the context of the BDF method.

while decreasing $\Delta t$ results in reductions of both the length of the relevant parabolic segment as well as the width of the parabola itself. Therefore, for $\Delta t>M_{t}$, increasing the number of grid points eventually causes some eigenvalues to enter the region of instability. But stability can be restored by a corresponding reduction in $\Delta t$; see Figure 4. In other words, a CFL-like condition of the form $\Delta t \leq F(h)(h=$ $2 \pi /(N+1))$ exists for $\Delta t>M_{t}$ : the "maximum stable $\Delta t$ " function $F(h)$ can be obtained by considering the intersection of the boundary locus of the BDF stability region (equation (50)) and the parabola $\Gamma_{m}$ with $m$ given by equation (56). It can be seen from the first line in equation (55) that, provided the coefficient of $\Delta t$ in the real part is much smaller than the corresponding coefficient of $\Delta t$ in the imaginary part then the CFL-like condition will be approximately linear around that point - as is apparent by consideration of the actual curves $\Delta t_{\max }=F(h)$ in Figure 5 near $h=1$. Of course, when $\Delta t$ is reduced to the value $M_{t}$ or below, then no increases in $N$ (reductions in $h$ ) result in instability - as may be appreciated by consideration of Figures 4 . We may thus emphasize: within the rectangular stability window no such CFL-like stability constraints exist.

For comparison, Figure 5 also displays the maximum stable $\Delta t$ curves for the Fourier-based Adams-Bashforth (AB) multistep methods of orders 3 and 4 as functions of the meshsize $h$ for the advection-diffusion equation under consideration with $\alpha=1$ and two values of $\beta$. We see that the stability of both the BDF and $\mathrm{AB}$ methods is controlled by an approximately linear CFL-type constraint of the form $\Delta t<C h$ for sufficiently large values of $h$. For smaller values of $h$ the CFL condition for the explicit method becomes more severe, and eventually reaches the approximately quadratic regime $\Delta t<C h^{2}$. By this point, the BDF methods have already entered the window of quasi-unconditional stability. For the particular value of $\alpha$ considered in these examples, at $h=\beta$ the maximum stable $\Delta t$ values for the BDF methods are approximately one hundred times larger than their $\mathrm{AB}$ counterparts. 
TABLE 2

Maximum stable $\Delta t$ values for the order-s BDF-ADI Navier-Stokes solvers introduced in Part I with $s=2, \ldots, 6$, in two spatial dimensions, and at Reynolds number $\mathrm{Re}=50$ and Mach number 0.8 , with various numbers $N_{y}$ of discretization points in the $y$ variable. The number of discretization points in the $x$ direction is fixed at $N_{x}=12$.

\begin{tabular}{c|ccccc}
\hline \hline & \multicolumn{5}{|c}{$s=\cdots$} \\
$N_{y}$ & 2 & 3 & 4 & 5 & 6 \\
\hline 12 & $6.1 \mathrm{e}-1$ & $3.5 \mathrm{e}-1$ & $9.1 \mathrm{e}-2$ & $4.1 \mathrm{e}-2$ & $1.7 \mathrm{e}-2$ \\
16 & $6.1 \mathrm{e}-1$ & $2.9 \mathrm{e}-1$ & $8.7 \mathrm{e}-2$ & $3.2 \mathrm{e}-2$ & $9.0 \mathrm{e}-3$ \\
24 & $6.1 \mathrm{e}-1$ & $1.3 \mathrm{e}-1$ & $5.9 \mathrm{e}-2$ & $1.9 \mathrm{e}-2$ & $5.3 \mathrm{e}-3$ \\
32 & $6.1 \mathrm{e}-1$ & $1.2 \mathrm{e}-1$ & $5.0 \mathrm{e}-2$ & $1.5 \mathrm{e}-2$ & $4.3 \mathrm{e}-3$ \\
48 & $6.1 \mathrm{e}-1$ & $1.0 \mathrm{e}-1$ & $4.2 \mathrm{e}-2$ & $1.3 \mathrm{e}-2$ & $3.7 \mathrm{e}-3$ \\
64 & $6.1 \mathrm{e}-1$ & $1.0 \mathrm{e}-1$ & $4.1 \mathrm{e}-2$ & $1.2 \mathrm{e}-2$ & $3.5 \mathrm{e}-3$ \\
96 & $6.1 \mathrm{e}-1$ & $1.0 \mathrm{e}-1$ & $4.0 \mathrm{e}-2$ & $1.2 \mathrm{e}-2$ & $3.1 \mathrm{e}-3$ \\
128 & $6.1 \mathrm{e}-1$ & $1.0 \mathrm{e}-1$ & $4.0 \mathrm{e}-2$ & $1.2 \mathrm{e}-2$ & $2.8 \mathrm{e}-3$ \\
\hline \hline
\end{tabular}

TABLE 3

Same as Table 3 but with Reynolds number $\mathrm{Re}=100$.

\begin{tabular}{c|ccccc}
\hline \hline & \multicolumn{5}{|c}{$s=\cdots$} \\
$N_{y}$ & 2 & 3 & 4 & 5 & 6 \\
\hline 12 & $6.4 \mathrm{e}-1$ & $3.4 \mathrm{e}-1$ & $5.9 \mathrm{e}-2$ & $3.4 \mathrm{e}-2$ & $1.5 \mathrm{e}-2$ \\
16 & $6.3 \mathrm{e}-1$ & $2.7 \mathrm{e}-1$ & $5.0 \mathrm{e}-2$ & $2.4 \mathrm{e}-2$ & $9.9 \mathrm{e}-3$ \\
24 & $6.3 \mathrm{e}-1$ & $1.1 \mathrm{e}-1$ & $4.5 \mathrm{e}-2$ & $1.9 \mathrm{e}-2$ & $6.1 \mathrm{e}-3$ \\
32 & $6.3 \mathrm{e}-1$ & $9.2 \mathrm{e}-2$ & $3.7 \mathrm{e}-2$ & $1.7 \mathrm{e}-2$ & $5.1 \mathrm{e}-3$ \\
48 & $6.3 \mathrm{e}-1$ & $7.8 \mathrm{e}-2$ & $3.2 \mathrm{e}-2$ & $1.6 \mathrm{e}-2$ & $4.6 \mathrm{e}-3$ \\
64 & $6.3 \mathrm{e}-1$ & $7.4 \mathrm{e}-2$ & $3.1 \mathrm{e}-2$ & $1.5 \mathrm{e}-2$ & $4.4 \mathrm{e}-3$ \\
96 & $6.3 \mathrm{e}-1$ & $7.2 \mathrm{e}-2$ & $3.0 \mathrm{e}-2$ & $1.5 \mathrm{e}-2$ & $4.3 \mathrm{e}-3$ \\
128 & $6.3 \mathrm{e}-1$ & $7.1 \mathrm{e}-2$ & $3.0 \mathrm{e}-2$ & $1.5 \mathrm{e}-2$ & $4.1 \mathrm{e}-3$ \\
\hline \hline
\end{tabular}

TABLE 4

Same as Table 2 but with Reynolds number $\mathrm{Re}=200$.

\begin{tabular}{c|ccccc}
\hline \hline & \multicolumn{5}{|c}{$s=\cdots$} \\
$N_{y}$ & 2 & 3 & 4 & 5 & 6 \\
\hline 12 & $5.5 \mathrm{e}-1$ & $2.9 \mathrm{e}-1$ & $4.5 \mathrm{e}-2$ & $2.8 \mathrm{e}-2$ & $1.3 \mathrm{e}-2$ \\
16 & $5.3 \mathrm{e}-1$ & $2.9 \mathrm{e}-1$ & $4.4 \mathrm{e}-2$ & $2.0 \mathrm{e}-2$ & $8.8 \mathrm{e}-3$ \\
24 & $5.5 \mathrm{e}-1$ & $1.1 \mathrm{e}-1$ & $2.5 \mathrm{e}-2$ & $1.3 \mathrm{e}-2$ & $4.6 \mathrm{e}-3$ \\
32 & $5.4 \mathrm{e}-1$ & $8.6 \mathrm{e}-2$ & $2.3 \mathrm{e}-2$ & $1.1 \mathrm{e}-2$ & $3.6 \mathrm{e}-3$ \\
48 & $5.3 \mathrm{e}-1$ & $6.6 \mathrm{e}-2$ & $2.1 \mathrm{e}-2$ & $9.5 \mathrm{e}-3$ & $2.9 \mathrm{e}-3$ \\
64 & $5.3 \mathrm{e}-1$ & $6.1 \mathrm{e}-2$ & $2.1 \mathrm{e}-2$ & $8.2 \mathrm{e}-3$ & $2.9 \mathrm{e}-3$ \\
96 & $5.3 \mathrm{e}-1$ & $5.9 \mathrm{e}-2$ & $2.1 \mathrm{e}-2$ & $8.3 \mathrm{e}-3$ & $2.4 \mathrm{e}-3$ \\
128 & $5.3 \mathrm{e}-1$ & $5.8 \mathrm{e}-2$ & $2.1 \mathrm{e}-2$ & $8.2 \mathrm{e}-3$ & $2.5 \mathrm{e}-3$ \\
\hline \hline
\end{tabular}

Clearly, the BDF methods are preferable in regimes where the $\mathrm{AB}$ methods suffer from the severe $\Delta t<C h^{2}$ CFL condition.

6. Quasi-unconditional stability for the full Navier-Stokes equations: A numerical study. Tables 2,3 , and 4 display numerically estimated maximum stable $\Delta t$ values for the Chebyshev-based BDF-ADI algorithm introduced in Part I for the full Navier-Stokes equations in two-dimensional space for various numbers of Chebyshev discretization points. (In contrast to the Fourier discretizations assumed in the previous sections, Chebyshev spatial discretizations are used in the present section in order to accommodate the nonperiodic boundary conditions that often arise in the Navier-Stokes context.) The specific problem under consideration is posed in the unit 
square $[0,1] \times[0,1]$ with Mach number 0.9 and various Reynolds numbers, with initial condition given by $\mathbf{u}=0, \rho=T=1$, and with a source term of the form

$$
f(x, y, t)=A \sin (2 \pi t) \exp \left(-\frac{1}{2 \sigma^{2}}\left(\left(x-x_{0}\right)^{2}+\left(y-y_{0}\right)^{2}\right)\right)
$$

as the right-hand side of the $x$ coordinate of the momentum equation $(A=6.0$, $\sigma^{2}=0.05$, and $\left.x_{0}=y_{0}=0.5\right)$. No-slip isothermal boundary conditions $(\mathbf{u}=0$, $T=1$ ) are assumed at $y=0$ and $y=1$, and a sponge layer (see Part I) of thickness 0.1 and amplitude 2.0 is enforced at $x=0$ and $x=1$. The algorithm was determined to be stable for a given $\Delta t$ if the solution does not blow up (i.e., the pointwise solution values do not exceed the value $10^{3}$ ) for 20000 time steps or for the number of time steps required to exceed $t=100$, whichever is greater. As indicated by the fully converged solution values produced by the solver, in the present example the "blowup" threshold $10^{3}$ should be much larger than any true values ever reached by the exact solution.

Tables 2, 3, and 4 suggest that the BDF-ADI Navier-Stokes algorithm introduced in Part I is indeed quasi-unconditionally stable. In particular, consideration of the tabulated values indicates that the BDF-ADI methods may be particularly advantageous whenever the timesteps required for stability in a competing explicit scheme for a given spatial discretization is much smaller than the timestep required for adequate resolution of the time variation of the solution.

7. Summary and conclusions. A variety of studies were put forth in this paper concerning the stability properties of the compressible Navier-Stokes BDFADI algorithms introduced in Part I, including rigorous stability proofs for associated BDF- and BDF-ADI-based algorithms for related linear equations, and numerical stability studies for the fully nonlinear problem. In particular, the present paper presents proofs of unconditional stability or quasi-unconditional stability for BDFADI schemes as well as certain associated unsplit BDF schemes, for a variety of diffusion and advection-diffusion linear equations in one, two, and three dimensions, and for schemes of orders $2 \leq s \leq 6$ of temporal accuracy. (The very concept of quasiunconditional stability was introduced in Part I to describe the observed stability character of the Navier-Stokes BDF-ADI algorithms introduced in that paper.) A set of numerical experiments presented in this paper for the compressible Navier-Stokes equation suggests that the algorithms introduced in Part I do enjoy the claimed property of quasi-unconditional stability.

\section{Appendix A. Proof of Theorem 4.1.}

Proof. Taking the inner product of equation (29) with $u$ we obtain

$$
\begin{aligned}
0 & =(u, \widehat{D} u)+(u, A u)+(u, B u)+b(u, A B D u) \\
& =(I)+(I I)+(I I I)+(I V),
\end{aligned}
$$

where $(I)=(u, \widehat{D} u),(I I)=(u, A u)$, etc. Our goal is to express the real part of the right-hand side in (62) as a sum of non-negative terms and telescoping terms of the form $D f$ for some non-negative numerical time series $f$. To that end, we consider the terms $(I)$ through $(I V)$ in turn.

(I) Using the expression (23) for $\widehat{D} u$ we obtain

$$
(I)=\frac{1}{2}(u, D u)+\frac{1}{2}(u, D \widetilde{w}),
$$


where $\widetilde{w}$ denotes the time series obtained by shifting $\widetilde{u}$ forwards by one timestep:

$$
\widetilde{w}=\left\{\widetilde{w}^{n}=\widetilde{u}^{n+1}: n \geq 1\right\} .
$$

To reexpress (63) we first note that for any two grid functions $a$ and $b$ we have the relation

$$
\begin{gathered}
|a-b|^{2}=|a|^{2}+|b|^{2}-2 \Re(a, b) \\
\Longrightarrow \Re(a, b)=\frac{1}{2}\left(|a|^{2}+|b|^{2}-|a-b|^{2}\right) .
\end{gathered}
$$

Therefore, for any time series $g$ we have

$$
\begin{aligned}
\Re(u, D g)^{n} & =\Re\left(u^{n}, g^{n}\right)-\Re\left(u^{n}, g^{n-1}\right) \\
& =\frac{1}{2}\left(\left|u^{n}\right|^{2}+\left|g^{n}\right|^{2}-\left|u^{n}-g^{n}\right|^{2}\right)-\frac{1}{2}\left(\left|u^{n}\right|^{2}+\left|g^{n-1}\right|^{2}-\left|u^{n}-g^{n-1}\right|^{2}\right) \\
& =\frac{1}{2}\left(D\left|g^{n}\right|^{2}-\left|u^{n}-g^{n}\right|^{2}+\left|u^{n}-g^{n-1}\right|^{2}\right) .
\end{aligned}
$$

Letting $g=u$ and $g=\widetilde{w}$ in (65) we obtain

$$
\Re(u, D u)=\frac{1}{2}\left(D|u|^{2}+|D u|^{2}\right)
$$

and

$$
\Re(u, D \widetilde{w})=\frac{1}{2}\left(D|\widetilde{w}|^{2}-|D u|^{2}+\left|D^{2} u\right|^{2}\right) .
$$

Replacing (66) and (67) in (63) we obtain

$$
\Re(I)=\frac{1}{4} D\left(|u|^{2}+|\widetilde{w}|^{2}\right)+\frac{1}{4}\left|D^{2} u\right|^{2} .
$$

Note that this equation expresses $\Re(I)$ as the sum of a telescoping term and a positive term, as desired.

$(I I)$ and $(I I I)$ The operator $A$ is clearly skew-Hermitian since $\delta_{x}$ is. Therefore

$$
\begin{aligned}
& (I I)=(u, A u)=-(A u, u)=-\overline{(u, A u)} \\
\Longrightarrow & \Re(I I)=0 .
\end{aligned}
$$

The relation

$$
\Re(I I I)=\Re(u, B u)=0
$$

follows similarly, of course.

$(I V)$ Lemma A.1 below tells us that

$$
\Re(u, A B D u) \geq \frac{1}{4} D\left(|A u|^{2}+|B u|^{2}\right)-\frac{1}{8}\left|D^{2} u\right|^{2} .
$$

Substituting (68), (69), (70), and (71) into equation (62) (recalling $b=2 / 3$ ) and taking the real part we obtain

$$
0 \geq \frac{1}{4} D\left(|u|^{2}+|\widetilde{w}|^{2}\right)+\frac{1}{6}\left(|A u|^{2}+|B u|^{2}+\left|D^{2} u\right|^{2}\right),
$$

which is the sum of a telescoping term and a non-negative term. Multiplying by the number four and summing the elements of the above numerical time series from $m=2$ to $n$ completes the proof of the theorem.

Copyright (C) by SIAM. Unauthorized reproduction of this article is prohibited. 
The following lemma concerns the bound (71) used in the proof above.

Lemma A.1. Any solution of equation (29) satisfies (71).

Proof. Taking the inner product of (29) with $A D u$ (using the form (22) of $\widehat{D} u$ ) we obtain

$$
0=(D u, A D u)+\frac{1}{2}\left(D^{2} u, A D u\right)+(A u, A D u)+(B u, A D u)+b(A B D u, A D u) .
$$

Since $A$ and $B$ commute and since $B$ is skew-Hermitian (equation (17)) we have

$$
(B u, A D u)=-(u, A B D u)
$$

for the next-to-last term in (73). Therefore, equation (73) can be reexpressed in the form

$$
\begin{aligned}
(u, A B D u) & =(D u, A D u)+\frac{1}{2}\left(D^{2} u, A D u\right)+(A u, A D u)+b(A B D u, A D u) \\
& =(I)+(I I)+(I I) .
\end{aligned}
$$

We consider each term in (74) in turn.

(I) Since $A$ is skew-Hermitian it follows that the real part of this term vanishes:

$$
\begin{aligned}
& (I)=(D u, A D u)=-(A D u, D u)=-\overline{(D u, A D u)}=-\overline{(I)} \\
\Longrightarrow & \Re(I)=0 .
\end{aligned}
$$

(II) Using Young's inequality

$$
a b \leq \frac{r}{2} a^{2}+\frac{1}{2 r} b^{2}
$$

(which, as is easily checked, is valid for all real numbers $a$ and $b$ and for all $r>0$ ) together with the Cauchy-Schwarz inequality we obtain

$$
\begin{aligned}
\Re(I I) & =\frac{1}{2} \Re\left(D^{2} u, A D u\right) \\
& \geq-\frac{1}{2}\left|\left(D^{2} u, A D u\right)\right| \\
& \geq-\frac{1}{2}\left|D^{2} u\right||A D u| \\
& \geq-\frac{1}{2}\left(\frac{1}{4}\left|D^{2} u\right|^{2}+|A D u|^{2}\right) \\
& =-\frac{1}{8}\left|D^{2} u\right|^{2}-\frac{1}{2}|A D u|^{2} .
\end{aligned}
$$

(III) By the finite-difference product rule (25) we obtain

$$
\begin{aligned}
(I I I) & =(A u, D(A u)) \\
& =D(A u, A u)-(D A u, A u)+(D A u, D A u) \\
& =D|A u|^{2}-\overline{(I I I)}+|A D u|^{2} \\
\Longrightarrow \Re(I I I) & =\frac{1}{2} D|A u|^{2}+\frac{1}{2}|A D u|^{2} .
\end{aligned}
$$

Copyright $@$ ㅇ by SIAM. Unauthorized reproduction of this article is prohibited. 
( $I V$ ) Again using the fact that $B$ is skew-Hermitian and commutes with $A$ it follows that

$$
\begin{aligned}
& (I V)=b(B A D u, A D u)=-b(A D u, B A D u)=-\overline{(I V)} \\
\Longrightarrow & \Re(I V)=0 .
\end{aligned}
$$

Combining the real parts of equations $(74),(75),(77),(78)$, and (79), we obtain

$$
\Re(u, A B D u) \geq \frac{1}{2} D|A u|^{2}-\frac{1}{8}\left|D^{2} u\right|^{2} .
$$

An analogous result can be obtained by taking the inner product of equation (29) with $B D u$ instead of $A D u$ and following the same steps used to arrive at equation (80). The result is

$$
\Re(u, A B D u) \geq \frac{1}{2} D|B u|^{2}-\frac{1}{8}\left|D^{2} u\right|^{2} .
$$

The lemma now follows by averaging equations (80) and (81).

\section{Appendix B. Proof of Theorem 4.3.}

Proof. Taking the inner product of (36) with $u$ we obtain

$$
\begin{aligned}
0 & =(u, \widehat{D} u)+(u, L u)-\left(u, F D^{2} u\right)+b(u, A B D u) \\
& =(I)+(I I)+(I I I)+(I V),
\end{aligned}
$$

where $(I)=(u, \widehat{D} u),(I I)=(u, L u)$, etc. As in Theorem 4.1, we reexpress the above equation using telescoping and non-negative terms to obtain the desired energy bound.

The term $(I)$ already occurs in the proof of Theorem 4.1; there we obtained the relation

$$
\Re(I)=\frac{1}{4} D\left(|u|^{2}+|\widetilde{w}|^{2}\right)+\frac{1}{4}\left|D^{2} u\right|^{2},
$$

where $\widetilde{w}$ is defined in (64). The term $(I I)=|u|_{L}^{2}$, in turn, is non-negative (see equation (37)) and thus requires no further treatment. The remaining two terms are considered in what follows.

(III) This term presents the most difficulty, since $F$ is not positive semidefinite. In what follows the term (III) is reexpressed as a a sum of two quantities, the first one of which can be combined with a corresponding term arising from the quantity $(I V)$ to produce a telescoping term, and the second of which will be addressed towards the end of the proof by utilizing Lemma B.1 below.

Let $v$ denote the time series obtained by shifting $u$ backwards by one timestep:

$$
v=\left\{v^{n}=u^{n-1}: n \geq 1\right\}
$$

clearly we have

$$
D u=u-v \quad \text { and } \quad D^{2} u=D u-D v .
$$

Thus, using the finite difference product rule (25) and the second relation in (85) we obtain

$$
\begin{aligned}
(I I I) & =-(u, F D(D u))=-(u, D F(D u)) \\
& =-D(u, F D u)+(D u, F D u)-\left(D u, F D^{2} u\right) \\
& =-D(u, F D u)+(D u, F D v) .
\end{aligned}
$$

Copyright $@$ by SIAM. Unauthorized reproduction of this article is prohibited. 
Applying the Cauchy-Schwarz inequality and Young's inequality (76) with $r=6$ together with (40) we obtain

$$
\begin{aligned}
\Re(I I I) & \geq-D \Re(u, F D u)-|D u||F D v| \\
& \geq-D \Re(u, F D u)-3|D u|^{2}-\frac{1}{12}|F D v|^{2} \\
& \geq-D \Re(u, F D u)-3|D u|^{2}-\frac{1}{3}|D v|_{A B}^{2} .
\end{aligned}
$$

The last term in the above inequality will be combined with an associated expression in $(I V)$ below to produce a telescoping term.

(IV) Using the finite difference product rule (26) together with the fact that $A B$ is a Hermitian positive semidefinite operator, we obtain

$$
\begin{aligned}
\Re(I V) & =\frac{2}{3} \Re(u, A B D u)=\frac{2}{3} \Re(D u, A B u) \\
& =\frac{1}{3} D(u, A B u)+\frac{1}{3}(D u, A B D u) \\
& =\frac{1}{3} D|u|_{A B}^{2}+\frac{1}{3}|D u|_{A B}^{2}
\end{aligned}
$$

(see equation (39)). Substituting (83), (86), and (87) into equation (82), recalling equation (64) and taking real parts, we obtain

$$
\begin{aligned}
0 \geq & \frac{1}{4} D\left(|u|^{2}+|\widetilde{w}|^{2}\right)+\frac{1}{4}\left|D^{2} u\right|^{2}+|u|_{L}^{2}-D \Re(u, F D u)-3|D u|^{2} \\
& +\frac{1}{3}\left(|D u|_{A B}^{2}-|D v|_{A B}^{2}\right)+\frac{1}{3} D|u|_{A B}^{2} \\
= & D\left(\frac{1}{4}|u|^{2}+\frac{1}{4}|\widetilde{w}|^{2}+\frac{1}{3}|u|_{A B}^{2}+\frac{1}{3}|D u|_{A B}^{2}-\Re(u, F D u)\right) \\
& +|u|_{L}^{2}+\frac{1}{4}\left|D^{2} u\right|^{2}-3|D u|^{2} .
\end{aligned}
$$

Adding the time series (88) from $m=2$ to $n$ and using the identity $\widetilde{w}^{n}=\widetilde{u}^{n+1}$, we obtain

$$
\begin{aligned}
M_{1} \geq & \frac{1}{4}\left|u^{n}\right|^{2}+\frac{1}{4}\left|\widetilde{u}^{n+1}\right|^{2}+\frac{1}{3}\left|u^{n}\right|_{A B}^{2}+\frac{1}{3}\left|(D u)^{n}\right|_{A B}^{2}+\sum_{m=2}^{n}\left|u^{n}\right|_{L}^{2} \\
& +\frac{1}{4} \sum_{m=2}^{n}\left|\left(D^{2} u\right)^{n}\right|^{2}-3 \sum_{m=2}^{n}\left|(D u)^{m}\right|^{2}-\Re\left(u^{n}, F(D u)^{n}\right),
\end{aligned}
$$

where

$$
M_{1}=\frac{1}{4}\left|u^{1}\right|^{2}+\frac{1}{4}\left|\widetilde{u}^{2}\right|^{2}+\frac{1}{3}\left|u^{1}\right|_{A B}^{2}+\frac{1}{3}\left|(D u)^{1}\right|_{A B}^{2}-\Re\left(u^{1}, F(D u)^{1}\right) .
$$

Using Cauchy-Schwarz and Young's inequalities along with the parabolicity relation (40) and the fact that $F$ is a Hermitian operator, the last term $-\Re\left(u^{n}, F(D u)^{n}\right)$ in (89) is itself estimated as follows:

$$
\begin{aligned}
-\Re\left(u^{n}, F(D u)^{n}\right) & =-\Re\left(F u^{n},(D u)^{n}\right) \\
& \geq-\left|F u^{n}\right|\left|(D u)^{n}\right| \\
& \geq-\frac{1}{12}\left|F u^{n}\right|^{2}-3\left|(D u)^{n}\right|^{2} \\
& \geq-\frac{1}{3}\left|u^{n}\right|_{A B}^{2}-3\left|(D u)^{n}\right|^{2} .
\end{aligned}
$$

Copyright (c) by SIAM. Unauthorized reproduction of this article is prohibited. 
Equation (89) may thus be re-expressed in the form

$$
\begin{aligned}
\frac{1}{4}\left|u^{n}\right|^{2} & +\frac{1}{4}\left|\widetilde{u}^{n+1}\right|^{2}+\frac{1}{3}\left|(D u)^{n}\right|_{A B}^{2}+\sum_{m=2}^{n}\left|u^{n}\right|_{L}^{2}+\frac{1}{4} \sum_{m=2}^{n}\left|D^{2} u\right|^{2} \\
& \leq M_{1}+3\left|(D u)^{n}\right|^{2}+3 \sum_{m=2}^{n}\left|(D u)^{m}\right|^{2} .
\end{aligned}
$$

Finally, applying Lemma B.1 below to the last two terms on the right-hand side of equation (90), we obtain

$$
3\left|(D u)^{n}\right|^{2}+3 \sum_{m=2}^{n}\left|(D u)^{m}\right|^{2} \leq 3 M_{2},
$$

where the constant $M_{2}$ is given by equation (92) below, and the proof of the theorem is thus complete.

The following lemma, which provides a bound on sums of squares of the temporal difference $D u$, is used in the proof of Theorem 4.3 above.

Lemma B.1. The solution $u$ of the Fourier-based BDF2-ADI scheme (35) for equation (32) with initial conditions $u^{0}, u^{1}$ satisfies

$$
\left|(D u)^{n}\right|^{2}+\left|u^{n}\right|_{L}^{2}+\frac{1}{2}\left(\left|(D u)^{n}\right|_{A}^{2}+\left|(D u)^{n}\right|_{B}^{2}\right)+\sum_{m=2}^{n}\left|(D u)^{m}\right|^{2} \leq M_{2}
$$

for $n \geq 2$, where

$$
M_{2}=\left|(D u)^{1}\right|^{2}+\left|u^{1}\right|_{L}^{2}+\frac{1}{2}\left(\left|(D u)^{1}\right|_{A}^{2}+\left|(D u)^{1}\right|_{B}^{2}\right) .
$$

Proof. We start by taking the inner product of equation (36) with $D u$ to obtain

$$
\begin{aligned}
0 & =(D u, \widehat{D} u)+(D u, L u)-\left(D u, F D^{2} u\right)+b(D u, A B D u) \\
& =(I)+(I I)+(I I I)+(I V) .
\end{aligned}
$$

We now estimate each of the terms $(I)$ through $(I V)$ in turn; as it will become apparent, the main challenge in this proof lies in the estimate of the term $(I I I)$.

$(I)$ Using (22) and the finite difference product rule (26), $(I)$ can be expressed in the form

$$
\begin{aligned}
\Re(I) & =\Re\left(D u, D u+\frac{1}{2} D^{2} u\right) \\
& =|D u|^{2}+\frac{1}{4} D|D u|^{2}+\frac{1}{4}\left|D^{2} u\right|^{2} .
\end{aligned}
$$

(II) Using equation (26) we obtain

$$
\Re(I I)=\Re(D u, L u)=\frac{1}{2} D(u, L u)+\frac{1}{2}(D u, L D u) .
$$

Since $L=A+B+F$ we may write

$$
\Re(I I)=\frac{1}{2} D|u|_{L}^{2}+\frac{1}{2}|D u|_{A+B}^{2}+\frac{1}{2}(D u, F D u) .
$$

Copyright $@$ by SIAM. Unauthorized reproduction of this article is prohibited. 
The last term in this equation (which is a real number in view of the Hermitian character of the operator $F$ ) will be used below to cancel a corresponding term in our estimate of $(I I I)$.

(III) Using (84) together with the second equation in (85), (III) can be expressed in the form

$$
\begin{aligned}
(I I I) & =-\left(D u, F D^{2} u\right) \\
& =-\frac{1}{2}(D u, F D u)+\frac{1}{2}(D u, F D v)-\frac{1}{2}\left(D u, F D^{2} u\right) .
\end{aligned}
$$

The first term on the right-hand side of (96) will be used to cancel the last term in (95). Hence it suffices to obtain bounds for the second and third terms on the right-hand side of equation (96).

To estimate the second term in (96) we consider the relation

$$
\frac{1}{2}(D u, F D v)=\frac{1}{2} \gamma \Delta t\left(D u, \delta_{x} \delta_{y} D v\right)=-\frac{\gamma}{4} \Delta t\left(\delta_{x} D u, \delta_{y} D v\right)-\frac{\gamma}{4} \Delta t\left(\delta_{y} D u, \delta_{x} D v\right),
$$

which follows from the fact that $\delta_{x}$ and $\delta_{y}$ are skew-Hermitian operators. Taking real parts and applying the Cauchy-Schwarz and Young inequalities together with the parabolicity condition (33), we obtain

$$
\begin{aligned}
\frac{1}{2} \Re(D u, F D v) \geq & -\frac{\sqrt{\alpha \beta}}{2} \Delta t\left(\frac{1}{2} \sqrt{\frac{\alpha}{\beta}}\left|\delta_{x} D u\right|^{2}+\frac{1}{2} \sqrt{\frac{\beta}{\alpha}}\left|\delta_{y} D v\right|^{2}\right) \\
& -\frac{\sqrt{\alpha \beta}}{2} \Delta t\left(\frac{1}{2} \sqrt{\frac{\beta}{\alpha}}\left|\delta_{y} D u\right|^{2}+\frac{1}{2} \sqrt{\frac{\alpha}{\beta}}\left|\delta_{x} D v\right|^{2}\right) \\
= & -\frac{1}{4} \Delta t\left(\alpha\left|\delta_{x} D u\right|^{2}+\beta\left|\delta_{y} D u\right|^{2}\right)-\frac{1}{4} \Delta t\left(\alpha\left|\delta_{x} D v\right|^{2}+\beta\left|\delta_{y} D v\right|^{2}\right) \\
= & -\frac{1}{4}|D u|_{A+B}^{2}-\frac{1}{4}|D v|_{A+B}^{2} .
\end{aligned}
$$

To estimate third term in (96) we once again use the Cauchy-Schwarz and Young inequalities and we exploit the relation (40); we thus obtain

$$
\begin{aligned}
-\frac{1}{2} \Re\left(D u, F D^{2} u\right) & =-\frac{1}{2} \Re\left(F D u, D^{2} u\right) \\
& \geq-\frac{1}{6}|F D u|^{2}-\frac{3}{8}\left|D^{2} u\right|^{2} \\
& \geq-\frac{2}{3}|D u|_{A B}^{2}-\frac{3}{8}\left|D^{2} u\right|^{2} .
\end{aligned}
$$

Taking the real part of (96) and using equations (98) and (99) we obtain the relation

$$
\Re(I I I) \geq-\frac{1}{2} \Re(D u, F D u)-\frac{1}{4}|D u|_{A+B}^{2}-\frac{1}{4}|D v|_{A+B}^{2}-\frac{2}{3}|D u|_{A B}^{2}-\frac{3}{8}\left|D^{2} u\right|^{2},
$$

which, as shown below, can be combined with the estimates for $(I),(I I)$, and $(I V)$ to produce an overall estimate that consists solely of non-negative and telescoping terms, as desired.

$(I V)$ In view of (39) we see that $(I V)$ coincides with the $P$-seminorm of $D u$ with $P=A B$,

$$
\Re(I V)=(I V)=\frac{2}{3}|D u|_{A B}^{2}
$$

Copyright $@$ by SIAM. Unauthorized reproduction of this article is prohibited. 
This term is non-negative and it therefore does not require further treatment.

To complete the proof of the lemma we take real parts in equation (93) and we substitute (94), (95), (100), and (101); the result is

$$
\begin{aligned}
0 & \geq|D u|^{2}+\frac{1}{4} D|D u|^{2}-\frac{1}{8}\left|D^{2} u\right|^{2}+\frac{1}{2} D|u|_{L}^{2}+\frac{1}{4}|D u|_{A+B}^{2}-\frac{1}{4}|D v|_{A+B}^{2} \\
& =|D u|^{2}-\frac{1}{8}\left|D^{2} u\right|^{2}+D\left(\frac{1}{4}|D u|^{2}+\frac{1}{2}|u|_{L}^{2}+\frac{1}{4}|D u|_{A+B}^{2}\right) .
\end{aligned}
$$

The first two terms on the right-hand side can be bounded by expanding $\left|D^{2} u\right|^{2}$ and using Cauchy-Schwarz and Young's inequalities to obtain

$$
\begin{aligned}
|D u|^{2}-\frac{1}{8}\left|D^{2} u\right|^{2} & =|D u|^{2}-\frac{1}{8}|D u-D v|^{2} \\
& =|D u|^{2}-\frac{1}{8}\left(|D u|^{2}+|D v|^{2}\right)+\frac{1}{4} \Re(D u, D v) \\
& \geq|D u|^{2}-\frac{1}{8}\left(|D u|^{2}+|D v|^{2}\right)-\frac{1}{4}|D u||D v| \\
& \geq|D u|^{2}-\frac{1}{4}\left(|D u|^{2}+|D v|^{2}\right) \\
& =\frac{1}{2}|D u|^{2}+\frac{1}{4} D|D u|^{2} .
\end{aligned}
$$

Substituting this result into (102), we obtain

$$
0 \geq \frac{1}{2}|D u|^{2}+D\left(\frac{1}{2}|D u|^{2}+\frac{1}{2}|u|_{L}^{2}+\frac{1}{4}|D u|_{A+B}^{2}\right),
$$

which, as needed, is expressed as a sum of non-negative and telescoping terms. Adding the time series (108) from $m=2$ to $n$ yields the desired equation (91), and the proof is thus complete.

Remark B.2. It is interesting to point out that Lemma B.1 by itself implies a weak stability result that follows from equation (91) and the Cauchy-Schwarz inequality:

$$
\begin{aligned}
\left|u^{n}\right| & =\left|u^{1}+\sum_{m=2}^{n}(D u)^{m}\right| \\
& \leq\left|u^{1}\right|+\sum_{m=2}^{n}\left|(D u)^{m}\right| \\
& \leq\left|u^{1}\right|+\left(n \sum_{m=2}^{n}\left|(D u)^{m}\right|^{2}\right)^{\frac{1}{2}} \\
& \leq\left|u^{1}\right|+\sqrt{n M_{2}},
\end{aligned}
$$

Theorem 4.3 provides a much tighter energy estimate than (109), of course.

\section{REFERENCES}

[1] R. M. Beam and R. Warming, An implicit factored scheme for the compressible Navier-Stokes equations, AIAA J., 16 (1978), pp. 393-402.

[2] R. M. Beam And R. WARming, Alternating direction implicit methods for parabolic equations with a mixed derivative, SIAM J. Sci. Stat. Comput., 1 (1980), pp. 131-159. 
[3] H. Bijl, M. H. Carpenter, V. N. Vatsa, and C. A. Kennedy, Implicit time integration schemes for the unsteady compressible Navier-Stokes equations: Laminar flow, J. Comput. Phys., 179 (2002), pp. 313-329, https://doi.org/10.1006/jcph.2002.7059.

[4] O. P. BRuno And M. Cubillos, Higher-order in time "quasi-unconditionally stable" ADI solvers for the compressible Navier-Stokes equations in $2 D$ and $3 D$ curvilinear domains, J. Comput. Phys., 307 (2016), pp. 476-495, http://www.sciencedirect.com/science/article/ pii/S0021999115008281.

[5] G. G. DAhLQuist, A special stability problem for linear multistep methods, BIT Numer. Math., 3 (1963), pp. 27-43, https://doi.org/10.1007/BF01963532.

[6] J. Douglas JR. AND J. E. GUnN, A general formulation of alternating direction methods, Numerische Mathematik, 6 (1964), pp. 428-453, https://doi.org/10.1007/BF01386093.

[7] D. Forti ANd L. Dedè, Semi-implicit BDF time discretization of the Navier-Stokes equations with VMS-LES modeling in a high performance computing framework, Comput. Fluids, 117 (2015), pp. 168-182, https://doi.org/10.1016/j.compfluid.2015.05.011.

[8] D. Gottlieb and J. S. Hesthaven, Spectral methods for hyperbolic problems, J. Comput. Appl. Math., 128 (2001), pp. 83-131.

[9] J. S. Hesthaven, S. Gottlieb, And D. Gottlieb, Spectral Methods for Time-Dependent Problems, Cambridge Monographs on Applied and Computational Mathematics 21, Cambridge University Press, Cambridge, 2007.

[10] S. KaraA, A hybrid Padé ADI scheme of higher-order for convection-diffusion problems, Int. J. Numer. Methods in Fluids, 64 (2010), pp. 532-548, http://onlinelibrary.wiley.com/doi/ 10.1002/fld.2160/abstract.

[11] D. A. Kopriva, Implementing spectral methods for partial differential equations: Algorithms for scientists and engineers, Springer, New York, 2009.

[12] J. D. LAmBerT, Numerical methods for ordinary differential systems: The initial value problem, John Wiley \& Sons, New York, 1991, http://dl.acm.org/citation.cfm?id=129839.

[13] R. LeVeque, Finite Difference Methods for Ordinary and Partial Differential Equations, Society for Industrial and Applied Mathematics, Philadelphia, 2007, http://epubs.siam.org/ doi/book/10.1137/1.9780898717839.

[14] D. W. Peaceman and H. H. Rachford, JR., The numerical solution of parabolic and elliptic differential equations, J. Soc. Ind. Appl. Math., 3 (1955), pp. 28-41, http://www.jstor.org/ stable/2098834.

[15] R. D. Richtmyer and K. W. Morton, Difference Methods for Initial-Value Problems, Interscience, New York, 1967.

[16] R. F. WARming AND R. M. Beam, An extension of A-stability to alternating direction implicit methods, BIT Numer. Math., 19 (1979), pp. 395-417, https://doi.org/10.1007/ BF01930993.

Copyright (C) by SIAM. Unauthorized reproduction of this article is prohibited. 